\title{
The Jieduan-Niwan (JDNW) Formula Ameliorates Hepatocyte Apoptosis: A Study of the Inhibition of E2FI-Mediated Apoptosis Signaling Pathways in Acute-on-Chronic Liver Failure (ACLF) Using Rats
}

\author{
Weixin $\mathrm{Hou}$ (D) ${ }^{1-4}$ \\ Yulin $\mathrm{HaO}^{1,2}$ \\ Wenlong Yang ${ }^{1,2}$ \\ Tian Tian ${ }^{1,2}$ \\ Peng Fang ${ }^{1,2}$ \\ Yuqiong $\mathrm{Du}^{1,2}$ \\ Lianyin Gao ${ }^{1,2}$ \\ Yanbin $\mathrm{Gao}^{3,4}$ \\ Qiuyun Zhang ${ }^{1,2}$
}

'Department of Hepatology, School of Traditional Chinese Medicine, Capital Medical University, Beijing, People's Republic of China; ${ }^{2}$ Department of Hepatology, Beijing Key Laboratory of Traditional Chinese Medicine Collateral Disease Theory Research, Capital Medical University, Beijing, People's Republic of China; ${ }^{3}$ Department of Endocrinology, School of Traditional Chinese Medicine, Capital Medical University, Beijing, People's Republic of China; ${ }^{4}$ Department of Endocrinology, Beijing Key Laboratory of Traditional Chinese Medicine Collateral Disease Theory Research, Capital Medical University, Beijing, People's Republic of China
Correspondence: Qiuyun Zhang

Department of Hepatology, School of

Traditional Chinese Medicine, Capital Medical

University, No. 10 You An Men Wai, Xi Tou

Tiao, Feng Tai District, Beijing, 100069, People's

Republic of China

Tel +86 $108391 \quad 1638$

Email 19970059@ccmu.edu.cn

Yanbin Gao

Department of Endocrinology, School of Traditional Chinese Medicine, Capital Medical University, No. 10 You An Men Wai, Xi Tou

Tiao, Feng Tai District, Beijing, 100069, People's Republic of China

Tel +86 1083911720

Email gyb@ccmu.edu.cn
Background: Acute-on-chronic liver failure (ACLF) is a severe, complicated human disease. E2F1-mediated apoptosis plays an important role in ACLF development. Jieduan-Niwan (JDNW) formula, a traditional Chinese medicine (TCM), has shown remarkable clinical efficacy in ACLF treatment. However, the hepatoprotective mechanisms of the formula are barely understood.

Purpose: This study aimed to investigate the mechanisms of JDNW formula in ACLF treatment by specifically regulating E2F1-mediated apoptotic signaling pathways in rats.

Methods: The JDNW components were determined by high-performance liquid chromatography (HPLC) analysis. The ACLF rat model was established using human serum albumin immune-induced liver cirrhosis, followed by D-galactosamine and lipopolysaccharide joint acute attacks. The ACLF rat was treated with JDNW formula. Prothrombin time activity was measured to investigate the coagulation function. Liver pathological injury was observed by hematoxylin-eosin (HE) and reticular fiber staining. The hepatocyte apoptosis index and apoptosis rate were determined by terminal deoxynucleotidyl transferase dUTP nick end labeling (TUNEL) assay and flow cytometry, respectively. Additionally, the expression of key genes and proteins that regulate E2F1-mediated apoptosis was analyzed by quantitative real-time PCR and Western blot.

Results: Seven major components of JDNW formula were detected. The formula ameliorated the coagulation function, decreased the hepatocyte apoptosis index and apoptosis rate, and alleviated liver pathological damage in ACLF rats. The down-regulation of the expression of genes and proteins from $\mathrm{p} 53$-dependent and non-p53-dependent apoptosis pathways and the up-regulation of the expression of genes from blocking anti-apoptotic signaling pathways indicated that JDNW formula inhibited excessive hepatocyte apoptosis in ACLF rats via E2F1-mediated apoptosis signaling pathways.

Conclusion: The findings indicate that JDNW formula protects livers of ACLF rats by inhibiting E2F1-mediated apoptotic signaling pathways, implying that these pathways might be a potential therapeutic target for ACLF treatment.

Keywords: traditional Chinese medicine, apoptosis, Jieduan-Niwan formula, acute-onchronic liver failure rat model, E2F1, pharmacological effect

\section{Introduction}

Acute-on-Chronic Liver Failure (ACLF) refers to liver failure manifestations such as acute jaundice and coagulation dysfunction caused by various inducements on the basis of chronic liver disease, which can be combined with multiple 
complications such as hepatic encephalopathy, ascites, infection, etc. ${ }^{1}$ It has such characteristics as rapid disease progression and high short-term mortality. ${ }^{2-4}$

The complex pathogenesis of ACLF is related to oxidative stress, inflammatory response, mitochondrial dysfunction, cell necrosis, and especially excessive apoptosis in liver cells. ${ }^{5-7}$ Apoptosis, generally considered a type of programmed cell death, plays a prominent role in maintaining cell homeostasis. However, excessive apoptosis results in ACLF when harmful stimuli are subjected to liver cells. ${ }^{8}$ The cell cycle-related transcription factor, E2F1, participates in the inducement of apoptosis, and the enhanced expression of E2F1 initiates a series of apoptotic processes.

The apoptosis signaling pathway is originated extensively by ligands binding to their death receptors in cells. For instance, the tumor necrosis factor- $\alpha$ (TNF- $\alpha$ ) combines with its receptor, the tumor necrosis factor receptor 1 (TNFR1), leading to the upregulation of the expression of E2F1 to induce apoptosis in cells. ${ }^{9,10}$ Three main E2F1mediated apoptosis pathways have been intensively studied: 1) the p53-dependent apoptosis pathway, 2) the nonp53-dependent apoptotic pathways, and 3) the blocking anti-apoptotic signal pathways. ${ }^{11}$

In the p53-dependent apoptosis pathway, E2F1 activates the expression of the transcripts of $p 14^{A R F}$, the inhibitor of the MDM2 ubiquitin ligase that hydrolyzes p53, to stabilize and accumulate p53. ${ }^{10,12-14}$ It has been reported that apoptotic peptidase activating factor 1 (APAF1) is a direct transcriptional target of $\mathrm{p} 53$, suggesting that the p53-dependent apoptosis pathway might relay on the upregulation of the expression of APAF1 to sensitize cells to apoptosis. ${ }^{15-17}$ Under the assistance of deoxyadenosine triphosphate (dATP) or ATP, APAF1 activates the initiator caspase Caspase-9, from the cysteinyl aspartate-specific proteinase family, by binding it at the N-terminal of APAF1 after interacting with cytochrome $c$, which is released from mitochondria in response to several apoptotic stimuli in the cytosol. ${ }^{10,17-19}$ The cleaved Caspase-9-/ $3 /-6 /-7$ are the active forms of Caspase-9/-3/-6/-7. The activated Caspase-9 (cleaved Caspase-9) subsequently initiates the downstream caspase-cascade system by activating the executioner Caspase-3. The activated Caspase-3 (cleaved Caspase-3) sequentially activates the other two executioners Caspase-6 and Caspase-7 to form cleaved Caspase- 6 and cleaved Caspase- 7 to finally induce apoptosis; cleaved Caspase-3 also activates Caspase-9 as feedback. ${ }^{10,17,19-21}$ These processes give rise to a caspase-activation pathway, namely the mitochondrionmediated pathway or the Caspase-9-dependent pathway. In addition, p53-induced miR34a expression may sensitize cells to apoptotic stimuli by decreasing B-cell lymphoma 2 (Bcl-2) levels. ${ }^{22}$

The p53-dependent apoptotic pathway is thought to be a vital approach of the E2F1-mediated apoptosis pathways, but not the unique pathway. It has been reported that E2F1 enhances the expression of the transcripts of $p 73$, which is the homologue of $p 53$, increases the expression of APAF1, activates Caspase-9, and initiates the downstream caspasecascade system to induce apoptosis in the absence of p53 and the mitochondria damage. ${ }^{10,15,17,23}$ Besides, E2F1 also directly induces the expression of APAF1 to directly activate Caspase- 9 without the accumulation of cytochrome $c,{ }^{17}$ and this process is concomitant with the initiation of a caspase-cascade for apoptosis. Both E2F1-mediated apoptosis pathways are called non-p53-dependent apoptotic pathways.

The TNF receptor-associated factor 2 (TRAF2) activates the NF-kB, which is the inhibitor of apoptosis, to release the anti-apoptotic factors in order to block the apoptosis initiated by the combination of TNF- $\alpha$ and TNFR1. But, the enhanced expression of E2F1 downregulates TRAF2 and inhibits the activation of NF-kB to block the anti-apoptotic signal pathway. ${ }^{18,24}$ Besides, the antiapoptotic proteins Bcl-2 and Mcl-1, a group of important mediators that control apoptosis, maintain the integrity of the mitochondrial membrane and prevent the release of cytochrome $c$, thereby indirectly regulating the activity of the caspase-cascade system in apoptosis. ${ }^{10,19,25-27}$ It has been reported that E2F1 potently represses the expression of Mcl-1 and Bcl-2 to provoke apoptosis. ${ }^{25,28}$ Both E2F1mediated apoptosis pathways belong to the blocking antiapoptotic signal pathways.

The pathogenesis of ACLF is complicated, and multiple organ failures are prone to occur. At present, there is still a lack of specific treatment measures. Early identification and treatment of predisposing factors are crucial. However, in up to $40 \%$ of patients the precipitating factor cannot be identified, ${ }^{29}$ making it difficult to prevent the disease from progressing. Extracorporeal liver support systems have shown potential for application in ACLF treatment, although no improvement in survival rate using molecular adsorbent recirculating systems was observed in subsequent studies. ${ }^{29}$ Liver transplantation is considered as the only effective treatment so far, ${ }^{2}$ however, it cannot be widely adopted due to the lack of supply 
sources, the high cost of the transplant, and the possible postoperative rejection. In China, traditional Chinese medicine (TCM) has been recruited as an important alternative and complementary treatment to ACLF for a long term because of its multiple targets and few side effects. Qian Ying, the Chinese well-known TCM doctor invents the Jieduan-Niwan (JDNW) method and formula, which are based on the long-term clinical experience and the pathogenesis theory of "toxin injuring hepatic body", especially the pathogenesis characteristics as the interaction between "toxin stasis" and "a deficiency of vital energy". Qian advocates that the treatment of ACLF should not only focus on detoxification and the promotion of blood circulation to remove blood stasis, and to reduce the development of the disease, but should also emphasize the tonification of the liver, spleen, and kidneys, in order to make the qi sufficient for powerful exorcism. At present, the JDNW method has been included in guidelines for clinical diagnosis and treatment of ACLF in TCM. ${ }^{30}$ The JDNW formula has been used in clinical practices for decades and exhibits remarkable efficacy in the amelioration of ACLF. ${ }^{31-33}$ The research group observed the efficacy of JDNW formula combined with western medicine in the treatment of 111 ACLF patients in the early stage. The results showed that it was significantly superior to the western medicine control group in terms of reducing mortality, and improving symptoms and liver function. ${ }^{33}$

Our previous studies demonstrated that the JDNW formula could increase the 24-h survival rate of ACLF rats, protect liver function, ${ }^{8}$ reduce the levels of inflammatory factors such as IL- 6 and TNF- $\alpha$ in serum and liver tissues, alleviate the damage to the liver nucleus, rough endoplasmic reticulum and mitochondria, protect the ultrastructure of liver cells, ${ }^{34}$ preventively inhibit hepatocyte apoptosis, ${ }^{8}$ and induce hepatocyte proliferation in ACLF rats. ${ }^{35,36}$ These results indicate the potential effectiveness of the JDNW formula in the prevention and treatment of ACLF.

Previous studies on ACLF had focused on inflammatory response ${ }^{34}$ and hepatocyte proliferation, ${ }^{35,36}$ as well as the prevention mechanism of ACLF regulation by the JDNW formula. ${ }^{8,35,36}$ However, the treatment mechanism of the JDNW formula for hepatocyte apoptosis via E2F1mediated apoptosis signaling pathways in ACLF is not fully understood. Therefore, this study was undertaken in an attempt to bridge this knowledge gap. An ACLF rat model was established, and confirmed by several histopathological experiments and coagulation function tests; the key elements involved in E2F1-mediated apoptotic signaling pathways were detected from gene and protein levels in order to reveal the underlying molecular mechanisms of the JDNW formula on ACLF rats.

\section{Materials and Methods \\ Drug Preparation}

The JDNW formula is composed of ten Chinese herbal medicines including Phyllanthus amarus Schumach. and Thonn, Astragalus membranaceus (Fisch.) Bunge, Trichosanthes kirilowii Maxim, Lysimachia christinae Hance, Viscum coloratum (Kom.) Nakai, Salvia miltiorrhiza Bunge, Rehmannia glutinosa (Gaertn.) DC, Aconitum carmichaeli Debx., Panax notoginseng (Burkill) F. H. Chen, and Curcuma phaeocaulis Valeton (Table 1). All the herbal medicines were purchased from Beijing TongRenTang (Group) Co. Ltd., Beijing, China. The proportion of the medicinal materials was 30:30:30:30:30:20:20:15:6:6. The medicinal materials were soaked in distilled water for 4-5 h. Aconitum carmichaeli Debx. was boiled first for $30 \mathrm{~min}$, and then other medicinal materials were added and all were boiled together for another $45 \mathrm{~min}$, after which the medicinal juice was poured out. The mixture of herbs was boiled for the second time for $30 \mathrm{~min}$ to collect the medicinal soup. In the end, the two times of water decoctions were mixed well to obtain the JDNW formula. According to the preliminary experimental results, the optimal dose of the JDNW formula for each rat was $21.7 \mathrm{~g} / \mathrm{kg} / \mathrm{d} ;{ }^{37}$ here the water decoction was concentrated at $4.34 \mathrm{~g} / \mathrm{mL}$ in a sterilized glass bottle and refrigerated at $4{ }^{\circ} \mathrm{C}$. It was used for the subsequent treatment of rats by gavage.

\section{Quantitative High-Performance Liquid Chromatography (HPLC) Analysis}

To prepare the samples for HPLC, 95\% ethanol was added into the filtered and concentrated JDNW solution $(4.34 \mathrm{~g} / \mathrm{mL})$; the concentration of ethanol was adjusted to $75 \%$ and the mixture left to stand overnight. The next day, the supernatant was taken, decompressed and dried at $60{ }^{\circ} \mathrm{C}$, and ground into powder. Dry powder of JDNW of mass $0.5163 \mathrm{~g}$ was taken into a $10 \mathrm{~mL}$ volumetric flask containing $5 \mathrm{~mL}$ of $70 \%$ ethanol and sonicated; then $70 \%$ ethanol was added to the sonicated mixture to $10 \mathrm{~mL}$ and filtered using a $0.45-\mu \mathrm{m}$ microporous membrane filter to harvest the JDNW testing solution. The standard substances catalpol $(\geq 98 \%$, B21678, Shanghai yuanye Bio-Technology Co., Ltd., 
Table I Constituents of the JDNW Formula

\begin{tabular}{|c|c|c|c|c|c|c|}
\hline Chinese Name & Botanical Name & Common Name & Genus & Family & $\begin{array}{l}\text { Weight } \\
\text { (g) }\end{array}$ & $\begin{array}{l}\text { Part } \\
\text { Used }\end{array}$ \\
\hline $\begin{array}{l}\text { Ku Wei Ye Xia } \\
\text { Zhu }\end{array}$ & $\begin{array}{c}\text { Phyllanthus amarus Schumach. \& } \\
\text { Thonn. }\end{array}$ & Carry Me Seed & Phyllanthus & Phyllanthaceae & 30 & Herb \\
\hline Huang Qi & $\begin{array}{c}\text { Astragalus membranaceus (Fisch.) } \\
\text { Bunge }\end{array}$ & Astragalus Root & Astragalus & Fabaceae & 30 & Root \\
\hline Gua Lou & Trichosanthes kirilowii Maxim & Chinese Cucumber & Trichosanthes & Cucurbitaceae & 30 & Fruit \\
\hline Jin Qian Cao & Lysimachia christinae Hance & Lysimachia & Lysimachia & Primulaceae & 30 & Herb \\
\hline Hu Ji Sheng & Viscum coloratum (Kom.) Nakai & Chinese Mistletoe & Viscum & Santalaceae & 30 & Stem, leaf \\
\hline Dan Shen & Salvia miltiorrhiza Bunge & Red Sage & Salvia & Lamiaceae & 20 & Root \\
\hline Di Huang & Rehmannia glutinosa (Gaertn.) DC & $\begin{array}{l}\text { Dried Rehmannia } \\
\text { Root }\end{array}$ & Rehmannia & Plantaginaceae & 20 & Root \\
\hline Fu Zi & Aconitum carmichaeli Debx. & Monkshood & Aconitum & Ranunculaceae & 15 & Root \\
\hline San $Q i$ & Panax notoginseng (Burkill) F. H. Chen & Notoginseng & Panax & Araliaceae & 6 & Root \\
\hline E Zhu & Curcuma phaeocaulis Valeton & Rhizoma Curcumae & Curcuma & Zingiberaceae & 6 & Rhizome \\
\hline
\end{tabular}

Abbreviation: JDNW formula, Jieduan-Niwan formula.

Shanghai, China), gallic acid (GA, $\geq 98 \%$, PS0258-0050, Chengdu Push Bio-Technology Co., Ltd., Chengdu, Sichuan, China), 3,4-dihydroxybenzaldehyde ( $\geq 98 \%$, B21613, Shanghai yuanye Bio-Technology Co., Ltd., Shanghai, China), chlorogenic acid (CA, $\geq 98 \%$, B20782, Shanghai yuanye Bio-Technology Co., Ltd., Shanghai, China), notoginsenoside R1 (NG-R1, $\geq 98 \%$, B21099, Shanghai yuanye Bio-Technology Co., Ltd., Shanghai, China), salvianolic acid B (Sal B, $\geq 98 \%$, B20261, Shanghai yuanye Bio-Technology Co., Ltd., Shanghai, China) and ginsenoside Rb1 (GRb1, $\geq 98 \%$, B21050, Shanghai yuanye Bio-Technology Co., Ltd., Shanghai, China) were precisely weighed and dissolved into methanol with their final concentrations at $10 \mathrm{mg} /$ $\mathrm{mL}, 2 \mathrm{mg} / \mathrm{mL}, 0.5 \mathrm{mg} / \mathrm{mL}, 2 \mathrm{mg} / \mathrm{mL}, 10 \mathrm{mg} / \mathrm{mL}$, $10 \mathrm{mg} / \mathrm{mL}$ and $10 \mathrm{mg} / \mathrm{mL}$, respectively. One hundred $\mu \mathrm{L}$ of each standard substance above was added to a 1-mL volumetric flask, diluted with methanol to the volume, and the dilution was named Standard No. 1. Five hundred $\mu \mathrm{L}$ of Standard No. 1 was transferred to a new 1-mL volumetric flask, diluted with methanol to the volume, called Standard No. 2. Similarly, Standard No. 3, 4, 5, and 6 were obtained by the dilution of 500 $\mu \mathrm{L}$ of the preceding Standard No., respectively, by up to $1 \mathrm{~mL}$ of methanol.
To control the quality and determine the constituents of the JDNW formula, HPLC analysis was performed to establish the fingerprint spectrum and Agilent 1260 series HPLC system (Agilent Technologies, Palo Alto, CA, USA) was utilized for chromatographic separation. The samples of the JDNW testing solution and standards were separated by Diamonsil ${ }^{\circledR} \mathrm{C} 18(2) \quad(250 \mathrm{~mm} \times$ $4.6 \mathrm{~mm}, 5 \mu \mathrm{m}$ ) columns (Dikma Technologies Inc., Beijing, China) at $35{ }^{\circ} \mathrm{C}$ and $0.1 \%$ phosphoric acid (A) and acetonitrile (B) were selected as the mobile phase. The gradient elution conditions were recorded as follows: $0-10 \mathrm{~min}, 3 \% \mathrm{~B} ; 10-20 \mathrm{~min}, 3-5 \% \mathrm{~B} ; 20-30$ min, 5-10\% B; 30-45 min, 10-13\% B; 45-65 min, $13-14 \%$ B; $65-80$ min, $14-15 \%$ B; $80-85$ min, $15-20 \%$ B; 85-125 min, $20-30 \% \mathrm{~B}$; $125-135 \mathrm{~min}, 30-50 \% \mathrm{~B}$; $135-140 \mathrm{~min}, 50-100 \% \mathrm{~B} ; 140-150 \mathrm{~min}, 100 \% \mathrm{~B}$. The flow rate was maintained at $1.0 \mathrm{~mL} / \mathrm{min}$, the injection volume was $10 \mu \mathrm{L}$, and the absorbance was recorded at $203 \mathrm{~nm}$. The HPLC results under the above conditions are shown in Figure S1. Briefly, the composition of catalpol, GA, 3,4-dihydroxybenzaldehyde, CA, NG-R1, Sal B, and GRb1 in the JDNW testing solution was $0.1324 \mathrm{mg} / \mathrm{mL}, \quad 0.0902 \mathrm{mg} / \mathrm{mL}, \quad 0.0073 \mathrm{mg} / \mathrm{mL}$, $0.0266 \mathrm{mg} / \mathrm{mL}, 0.1905 \mathrm{mg} / \mathrm{mL}, 0.5436 \mathrm{mg} / \mathrm{mL}$ and $0.1834 \mathrm{mg} / \mathrm{mL}$, respectively. 


\section{Animal Materials}

Male Wistar rats (weighing 180-200 g) were purchased from Beijing Vital River Laboratory Animal Technology Co., Ltd., Beijing, China (Certificate No. SCXK-JING 2016-0006) and were maintained at the Laboratory Animal Center of the Capital Medical University under specific-pathogen-free (SPF) conditions at $22.4 \pm 3.0{ }^{\circ} \mathrm{C}$, with humidity of $51.3 \pm 2.2 \%$ and a 12 -h light/12-h dark photoperiod.

\section{Establishment of ACLF Model and Treatment}

The male Wistar rats $(\mathrm{n}=109)$ were randomly divided into two groups, the normal group $(\mathrm{n}=9)$ and the treatment group $(\mathrm{n}=100)$. The treatment group was injected with human serum albumin (HSA) (Sigma, St Louis, MO, USA) to trigger liver cirrhosis. ${ }^{38}$ Rats in the treatment group were each sensitized immunologically by a subcutaneous injection of $0.5 \mathrm{~mL}$ HSA (containing a dose of $4 \mathrm{mg}$ ) per time on the 1st, 14th, 24th and 34 th $\mathrm{d}$. Then, the rats were injected with $2.5 \mathrm{mg}$ HSA through their tail veins, followed by a successive dosage increase of $0.5 \mathrm{mg}$ per time until a total dose of $4.5 \mathrm{mg}$ was reached for each rat. Subsequently, maintaining the dose of $4.5 \mathrm{mg}$, and the continued injection until the sixth week, the volume of each injection was $0.5 \mathrm{~mL}$. The rats were injected twice a week. Masson's trichrome staining was performed to confirm the occurrence of hepatic fibrosis from the liver biopsy of the treatment group rats (Figure S2). The rats with hepatic fibrosis stage III or above $(n=60)$ were selected and continued to be injected with $4.5 \mathrm{mg}$ HSA through their tail veins for 2 weeks to establish the cirrhosis model. According to Liu Xuhua's modeling method, ${ }^{39}$ the cirrhotic rats were intraperitoneally injected with D-galactosamine (400 mg/kg, Sigma, St Louis, MO, USA) and lipopolysaccharide $(100 \mu \mathrm{g} / \mathrm{kg}$, Sigma, St Louis, MO, USA) (D-GalN/LPS) for acute attacks to establish the ACLF model. Rats in the normal group were offered the same volume of $0.9 \%$ physiological saline at each time point. After establishing the ACLF model, the rats $(n=54)$ in the treatment group were randomly divided into two groups as the JDNW group and model group, and each group was randomly divided into three subgroups as JDNW/model group $(5 \mathrm{~d}, 10 \mathrm{~d}, 15 \mathrm{~d})(\mathrm{n}=9$ per group). At the optimal dose $(21.7 \mathrm{~g} / \mathrm{kg} / \mathrm{d})$ mentioned above,${ }^{37}$ the JDNW groups received continuous gavage of JDNW concentrated solution $(5 \mathrm{~mL} / \mathrm{kg} / \mathrm{d})$ for 5,10 and $15 \mathrm{~d}$ after acute attacks for $24 \mathrm{~h}$. The normal group and the model groups received gavage of isodose $0.9 \%$ physiological saline continuously at the same time points. The blood and liver samples of rats in each group were collected at 5,10 , and $15 \mathrm{~d}$ after the treatments for future analysis.

\section{Coagulation Condition}

Blood treated with sodium citrate was centrifuged at $3000 \mathrm{rpm}$ for $15 \mathrm{~min}$ at $4{ }^{\circ} \mathrm{C}$ to separate the plasma, and the prothrombin time activity (PTA) of each sample was detected using the Beckman Coulter ACL-TOP 700 Coagulation Analyzer (Beckman Coulter, Inc., Chaska, MN, USA) within $2 \mathrm{~h}$.

\section{Histological Examinations}

For hematoxylin and eosin staining (HE staining), the liver tissues were fixed with $10 \%$ formaldehyde solution and embedded in paraffin wax, then they were cut into 5 - $\mu \mathrm{m}$-thick slices and fixed on the slide with neutral gum to make paraffin sections. After wiping off paraffin from sections using xylene and alcohol, the sections were stained with hematoxylin dye and eosin dye. The results were observed with a Nikon Eclipse 80i light microscope $(\times 200)$ (Nikon, Tokyo, Japan).

For reticular fiber staining, the sections were soaked in $0.25 \%$ potassium permanganate solution after dewaxing, bleached with $2.5 \%$ oxalic acid solution, and dipped in $2 \%$ ammonium ferric sulfate solution and silver ammonia solution. After fixing with $2 \%$ sodium thiosulfate solution, the sections were stained with Ponceau S solution. The results were observed with a Nikon Eclipse 80i light microscope $(\times 100)$ (Nikon, Tokyo, Japan).

\section{Terminal Deoxynucleotidyl Transferase dUTP Nick End Labeling (TUNEL) Assay}

The hepatocyte apoptosis in paraffin sections was detected by the Colorimetric TUNEL Apoptosis Assay Kit (Beyotime Biotechnology, Shanghai, China), according to the manufacturer's instructions. The results were observed under the ZEISS Axio Imager A2 Upright Metallurgical Microscope $(\times 200$ and $\times 400)($ Carl Zeiss Meditec, Jena, Thuringia, Germany). Three sections were randomly selected from each group, five fields were randomly selected from each section under the high-power microscopic field $(\times 400)$, and 100 hepatocytes were counted in 
each field. The apoptosis index=the number of apoptotic cells/total number of cells $\times 100 \%$.

\section{Flow Cytometry Assay}

The liver samples of rats were harvested before blood collection. Briefly, liver tissues of equal sizes were taken from the same part of the left lobe of the rat livers and rapidly placed in a 1640 medium containing 5\% fetal bovine serum (FBS). The liver tissues were moved from the medium onto a 300-mesh filter and ground gently to let the cells pass through the filter. Afterward, the cells were collected and washed with phosphate buffer saline (PBS). $2 \times 10^{6}$ cells were placed in the flow tube and washed with PBS. Then, $1 \mu \mathrm{L}$ Annexin V YO-PRO- 1 dye and $1 \mu \mathrm{L}$ PI dye from Vybrant ${ }^{\circledR}$ Apoptosis Assay Kit \#4 (Invitrogen, Carlsbad, CA, USA) were added to the flow tubes. The cells were static-cultured at $4{ }^{\circ} \mathrm{C}$ in the dark for $20 \mathrm{~min}$ and resuspended in PBS. Finally, the hepatocyte apoptosis rate was detected by flow cytometry-BD FACS Canto II Plus (BD Biosciences, San Jose, CA, USA).

\section{Quantitative Real-Time PCR (RT-qPCR) Analysis}

The total RNAs were extracted from the liver tissues using the RNAprep pure Tissue Kit (TIANGEN Biotech Co., Ltd., Beijing, China), according to the manufacturer's instructions, and were reverse-transcribed into cDNA using the FastKing RT Kit (With gDNase) (TIANGEN Biotech Co., Ltd., Beijing, China), again according to the manufacturer's instructions. The RT-qPCR reaction system was established by SuperReal PreMix Plus (SYBR Green) Kit (TIANGEN Biotech Co., Ltd., Beijing, China), according to the manufacturer's instructions, with $\beta$-actin as the internal control, at the following conditions: $95{ }^{\circ} \mathrm{C}$ for 15 min, 40 cycles of $95{ }^{\circ} \mathrm{C}$ for $10 \mathrm{~s}, 60^{\circ} \mathrm{C}$ for $20 \mathrm{~s}$ and $72{ }^{\circ} \mathrm{C}$ for $30 \mathrm{~s}$. Finally, gene expression was statistically analyzed with the $2^{-\Delta \Delta C T}$ method. All primer sequences for the RTqPCR analyses are presented in Table S1.

\section{Western Blotting Analysis}

The liver tissues were lysed with RIPA lysis buffer containing protease and phosphatase inhibitors, and total proteins were collected after homogenization and centrifugation. The concentration of proteins was determined by BCA Protein Assay Kit (Applygen Technologies Inc., Beijing, China), according to the manufacturer's instructions, and adjusted to a final concentration of $3 \mu \mathrm{g} / \mu \mathrm{L}$. Ten percent
SDS polyacrylamide gel electrophoresis (SDS-PAGE) was used to separate the proteins, which were then transferred to polyvinylidene difluoride (PVDF) membranes (Bio-Rad, Hercules, CA, USA). The membranes were placed in 5\% skimmed milk powder, sealed for $1 \mathrm{~h}$, and then incubated with a diluted solution of primary anti-E2F1 (1:500, sc-251, Santa Cruz Biotechnology, Santa Cruz, CA, USA), antiCaspase-3 (1:1000, \#9662, Cell Signaling Technology, Danvers, MA, USA), anti-Caspase-6 (1:1000, 101981-AP, Proteintech Group, Inc., Chicago, IL, USA), antiCaspase-7 (1:1000, \#9492, Cell Signaling Technology, Danvers, MA, USA), anti-Caspase-9 (1:1000, ab184786, Abcam, Cambridge, UK), anti- $\beta$-actin (1:5000, A0101, LabLead Bio-Technology Co., Ltd., Beijing, China), or anti-GAPDH (1:5000, G0100, LabLead Bio-Technology Co., Ltd., Beijing, China), respectively, overnight at $4{ }^{\circ} \mathrm{C}$. Anti- $\beta$-actin or anti-GAPDH was utilized as an internal control based on the molecular weight of the detected proteins. After washing four times with $1 \times$ Tris-bufferedsaline with Tween (TBST), the membranes were incubated with anti-mouse secondary antibody (1:5000, S0100, LabLead Bio-Technology Co., Ltd., Beijing, China) or anti-rabbit secondary antibody (1:5000, S0101, LabLead Bio-Technology Co., Ltd., Beijing, China) at room temperature for $1 \mathrm{~h}$. The protein bands were visualized using the Super ECL Plus Detection Reagent (Applygen Technologies Inc., Beijing, China), according to the manufacturer's instructions. The blot intensities were quantified with Image $\mathrm{J}^{40}$ and compared with an internal control $(\beta$ actin antibody or GAPDH antibody) to determine the differences in protein expression in each group. All the experiments were done in triplicate.

\section{Statistical Analyses}

SPSS 20.0 (SPSS Inc., Chicago, IL, USA) software was used for statistical analysis of experimental data. QQ plot (Figure S3) together with Shapiro-Wilk test were used to test the normality of experimental data. Statistical comparison among multiple groups was carried out by one-way ANOVA followed by the least significant difference (LSD) test (data are normally distributed and homogeneity of variance, results were expressed as mean \pm standard deviation (SD)) or Kruskal-Wallis test followed by Dunn's test (data are nonnormally distributed or without homogeneity of variance, results were expressed as the median (min - max)). $P$ value denoted the significant difference, $P<0.05$ indicating statistical significance and $P<0.01, P<0.001$, and $P<0.0001$ implying notable statistical significance. GraphPad Prism 8 
(GraphPad Software, Inc., La Jolla, CA, USA) was used for plotting the graphs.

\section{Results}

\section{The JDNW Formula Ameliorated the Coagulation Function of ACLF Rats}

The PTA of rats in normal, model and JDNW groups were measured to detect whether the JDNW formula could ameliorate the coagulation function of ACLF rats (Figure 1). The result showed that, compared with the normal group, the PTA of rats in the model groups decreased significantly at each time point ( $5 \mathrm{~d}, 10 \mathrm{~d}, 15 \mathrm{~d}$ ), with a gradually decreasing trend over the entire time period (each $P<0.0001$ ). It suggested that the ACLF rats had coagulation dysfunction, which is consistent with the clinical manifestations of ACLF patients. Compared with the model groups, the PTA of rats in the JDNW groups increased significantly at the corresponding time points $(P=0.0415, P<0.0001, P<0.0001)$. These results indicated that the JDNW formula ameliorated the coagulation function of ACLF rats.

\section{The JDNW Formula Ameliorated the Pathological Injury of ACLF Rats}

To examine whether the JDNW formula could alleviate the pathological injury of ACLF rats, the liver tissues in normal, model, and JDNW groups were detected by HE staining and reticular fiber staining. In the results of $\mathrm{HE}$ staining, the hepatic lobules were intact in the normal group, with no denatured or necrotic cells. The liver cells in the model groups presented edema, deformation, necrosis, and inflammatory cell infiltration. Liver fibrosis was

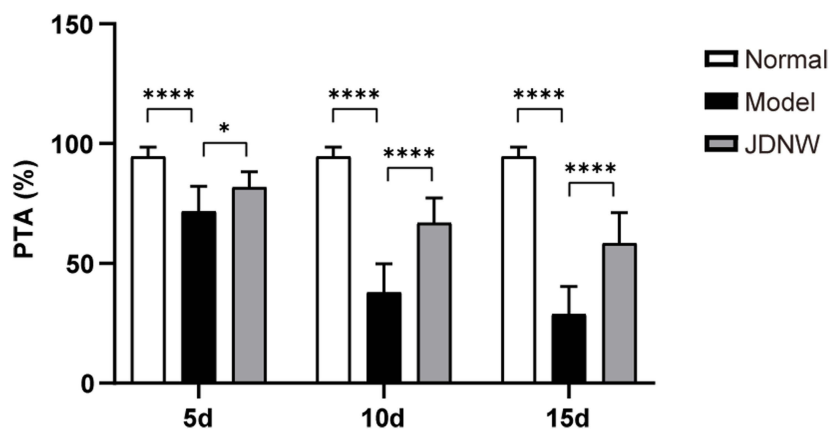

Figure I The JDNW formula ameliorated the coagulation function of ACLF rats. Notes: PTA of rats in normal, model and JDNW groups was measured by a coagulation analyzer; the results were expressed as mean $\pm S D$, asterisks denote the significance levels: $* * * * P<0.0001, * P<0.05$.

Abbreviations: JDNW, Jieduan-Niwan; ACLF, acute-on-chronic liver failure; PTA, prothrombin time activity; SD, standard deviation. obvious, the pseudolobules were widely formed, and some liver tissues showed massive or submassive necrosis and obvious bleeding. The severity of the pathological phenomena was aggravated over time. Interestingly, the typical pathological damage of the ACLF rats was alleviated after treatment with the JDNW formula. In the JDNW groups, the degree of inflammatory cell infiltration and tissue necrosis decreased compared with the model groups (Figure 2A). Besides, the results of reticular fiber staining indicated that there was no obvious abnormal fiber formation in the normal group. But in the model groups, liver fibrosis was distinct. The reticular fibers were thick and numerous in the portal area, and the degree of liver fibrosis gradually increased over time; pseudolobules were formed, the hepatic cords were disorderly and the reticular scaffolds collapsed. Remarkably, although there were reticular fibers and pseudolobules in the JDNW groups, the severity of hepatic fibrosis was lightened at the corresponding time points compared with the model groups (Figure 2B). These results indicated that the ACLF model was established successfully and the JDNW formula ameliorated the pathological damage to liver tissues in ACLF rats.

\section{The JDNW Formula Reduced the Hepatocyte Apoptosis Index and Apoptosis Rate of ACLF Rats}

To examine whether the JDNW formula could reduce the hepatocyte apoptosis level of ACLF rats, the hepatocyte apoptosis index and apoptosis rate were measured. TUNEL assay was employed to measure the apoptosis index (Figure 3A) and negative controls were shown in Figure S4. The results showed that TUNEL positive cells with brown nuclei were barely detected in the normal group. In contrast, a large number of TUNEL positive cells appeared in the model groups, and the apoptosis index showed a gradually increasing trend over time (each $P<0.0001$ ). Interestingly, the TUNEL positive cells and apoptosis index of the JDNW formula-treated ACLF rats significantly reduced at each corresponding time point $(P=0.0019, P<0.0001, P<0.0001)$. Besides, flow cytometry was used to detect the hepatocyte apoptosis rate (Figure 3B). The results showed that, compared with the normal group, the hepatocyte apoptosis rate in the model groups significantly increased on the 5th, 10th and 15th $\mathrm{d}$, with a gradually increasing trend over the entire time period $(P=0.0091, P=0.0001, P<$ 


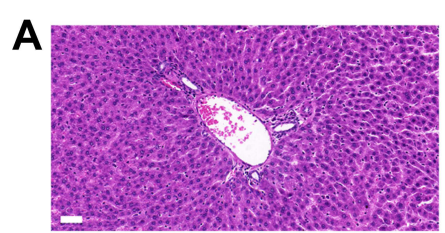

Normal

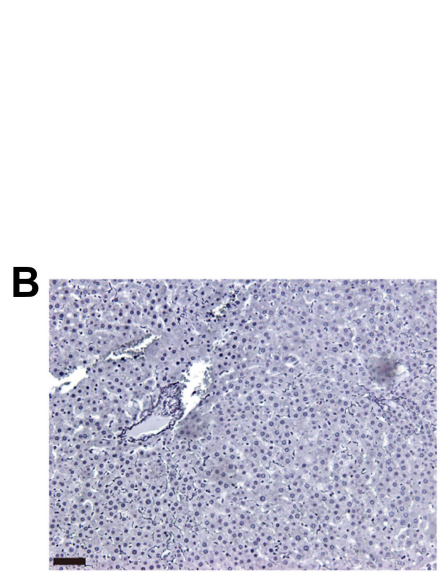

Normal

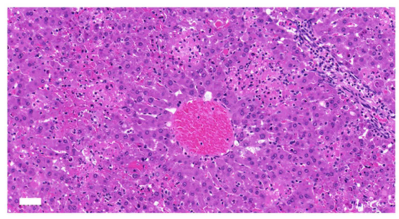

Model on $5 \mathrm{~d}$

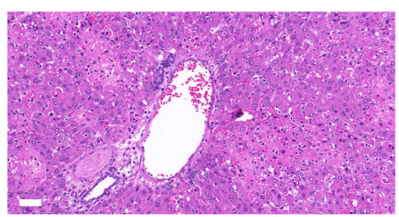

JDNW on $5 \mathrm{~d}$

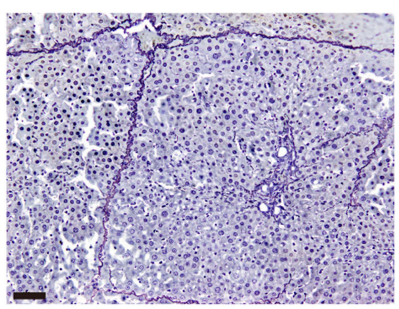

Model on $5 \mathrm{~d}$

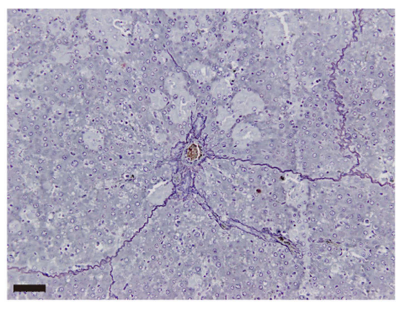

JDNW on $5 d$

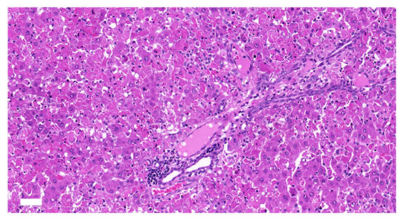

Model on $10 \mathrm{~d}$

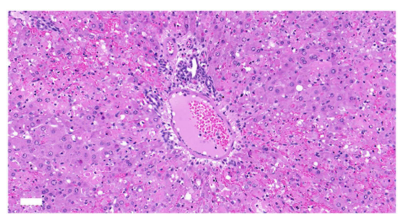

JDNW on $10 d$

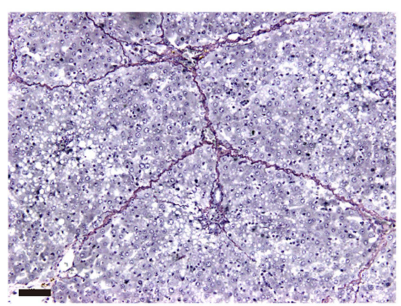

Model on $10 \mathrm{~d}$

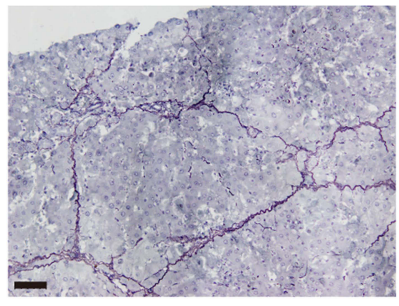

JDNW on $10 d$

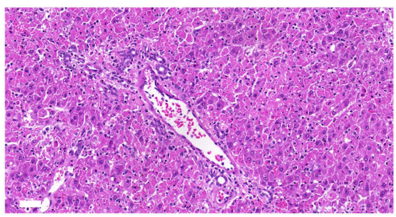

Model on 15 d

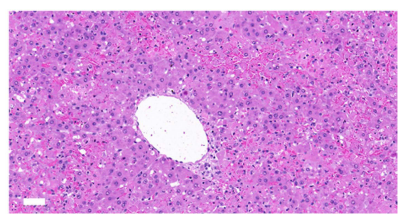

JDNW on 15 d

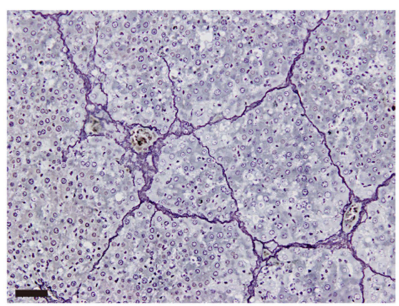

Model on $15 d$

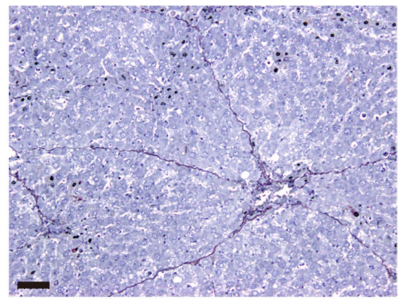

JDNW on 15 d

Figure 2 The JDNW formula ameliorated the pathological injury of ACLF rats.

Notes: (A) HE staining of hepatic tissues from the different groups (magnification, $\times 200$ ). Scale bars in all images are $50 \mu \mathrm{m}$. (B) Reticular fiber staining of hepatic tissues from the different groups (magnification, $\times 100$ ). Scale bars in all images are $100 \mu \mathrm{m}$.

Abbreviations: JDNW, Jieduan-Niwan; ACLF, acute-on-chronic liver failure; HE staining, hematoxylin and eosin staining.

0.0001). Notably, compared with the model groups, the apoptosis rate of the JDNW groups decreased at each time point, and the most significant decrease occurred on the 15 th $\mathrm{d}(P=0.0491)$. Taken together, these results indicated that the JDNW formula significantly reduced the hepatocyte apoptosis index and apoptosis rate, thereby inhibiting excessive apoptosis of hepatocytes protecting hepatocytes from ACLF in rats.

\section{The JDNW Formula Down-Regulated the Expression of TNFRI and E2FI to Protect Hepatocytes in ACLF Rats}

To investigate the regulatory effect of the JDNW formula on TNFR1 and E2F1, RT-qPCR was employed to monitor the transcript levels of TNRF1 (Figure 4A) and Western blot was used to detect the expression of E2F1 (Figure 4B). The results indicated that, compared with the normal group, the expression of TNFR1 and E2F1 in the model groups increased significantly on the 5th, 10th and 15th d TNFR1: $P=0.3445, P=0.0464, P=0.0001$; E2F1: $P=0.0001, P<0.0001, P<0.0001)$. Compared with the model groups, the expression of TNFR1 and E2F1 decreased significantly in the JDNW groups at each corresponding time point, and the most significant down-regulation of TNFR1 transcripts occurred on the 10th and 15th $\mathrm{d}(P=0.0405, P=0.0099)$, while the most significant decrease in E2F1 was observed on the 5th, 10th and 15th $\mathrm{d}(P=0.0292, P<0.0001, P<0.0001)$. These findings indicated that the JDNW formula effectively down-regulated the expression of TNFR1 and E2F1 in order to protect hepatocytes in ACLF rats. 

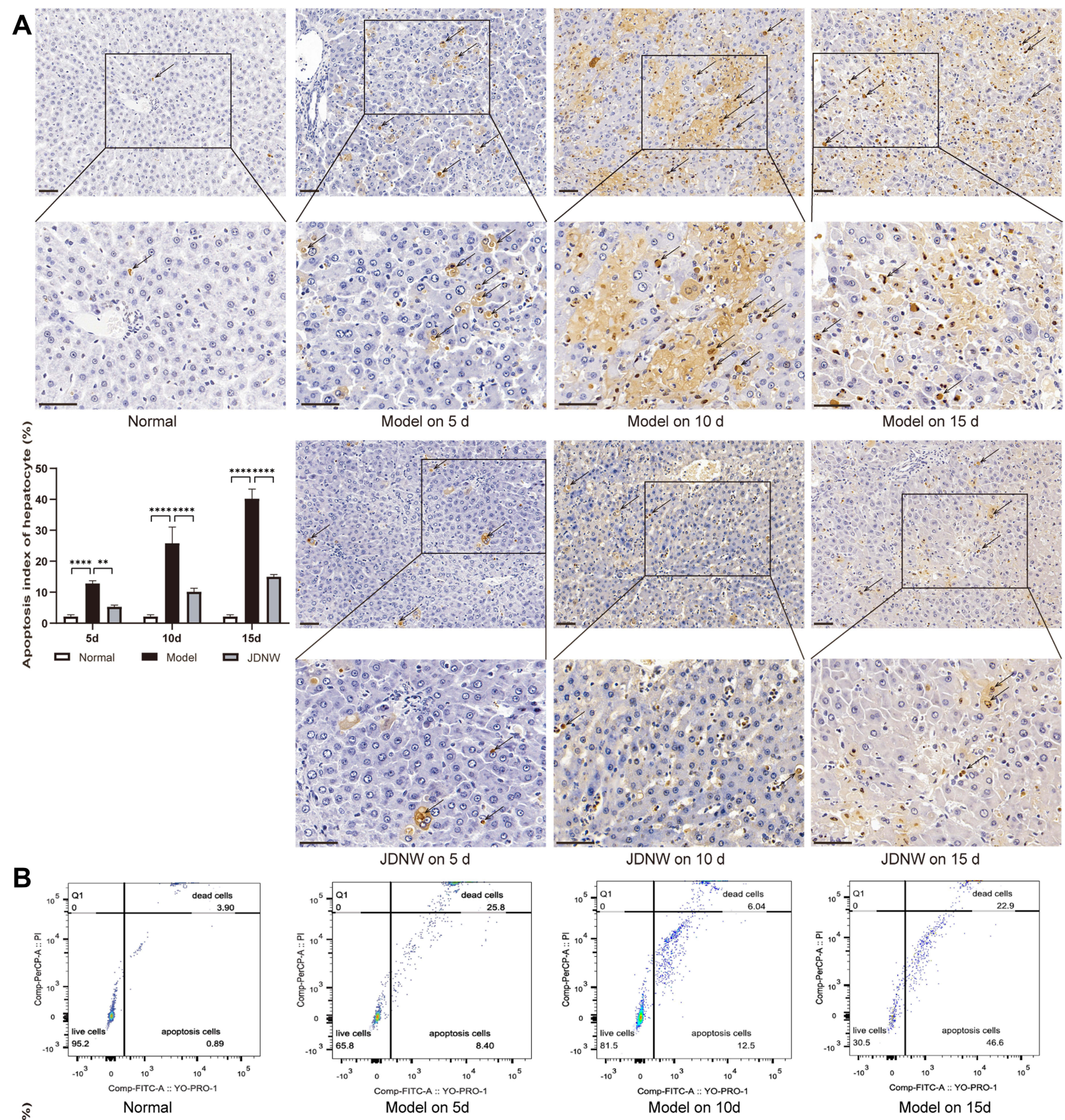

JDNW on $5 d$
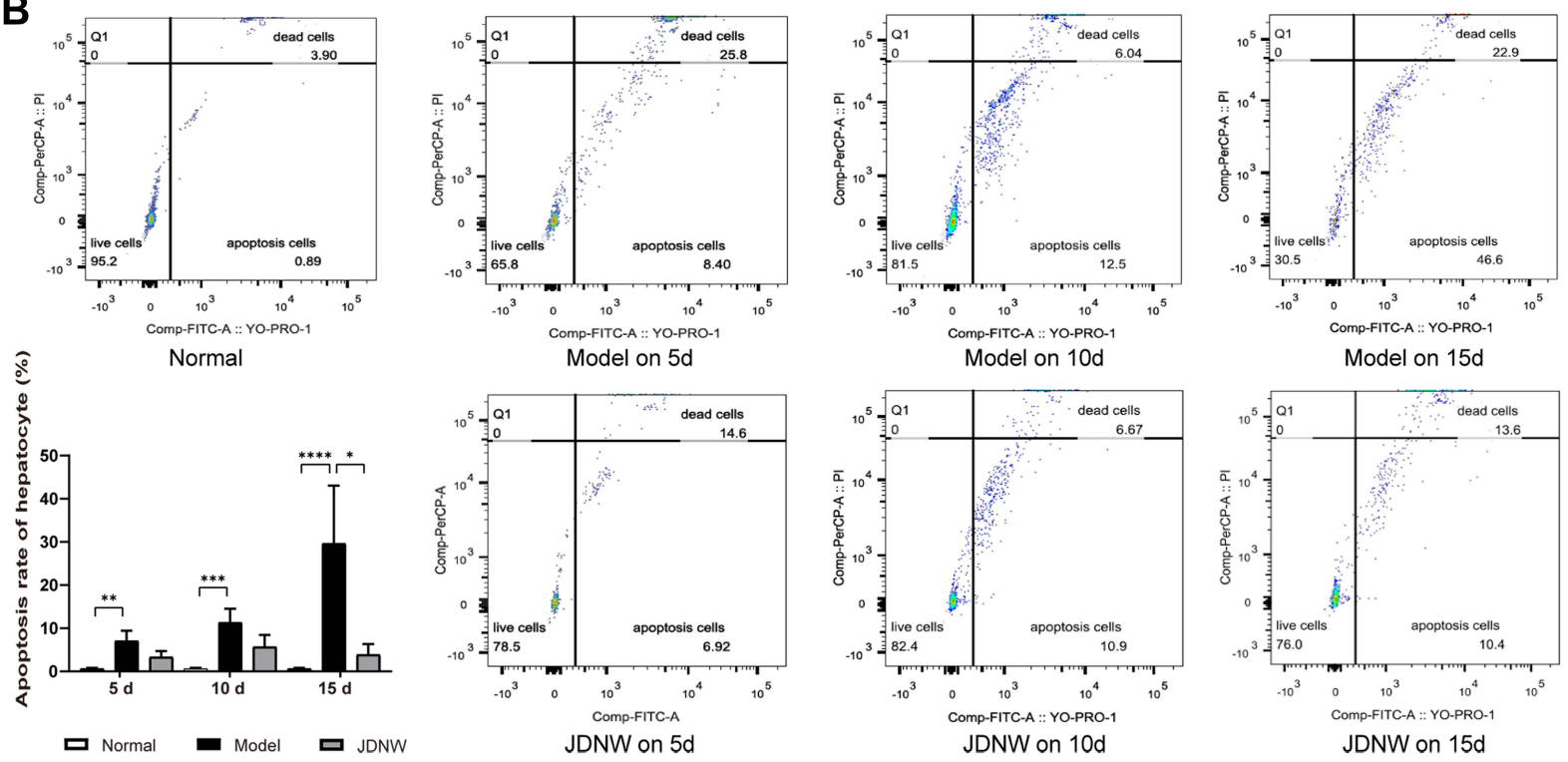

Figure 3 The JDNW formula reduced the hepatocyte apoptosis index and apoptosis rate of ACLF rats.

Notes: (A) TUNEL assay of hepatic tissues from the different groups (magnification, $\times 200$, up). The TUNEL positive cells are boxed in each group, and the boxed regions are further enlarged below (magnification, $\times 400$ ). Arrows indicate TUNEL positive cells. Scale bars in all images are $50 \mu \mathrm{m}$. The results were expressed as mean \pm SD. (B) Hepatocyte apoptosis rate in each group was determined by flow cytometry. The results were expressed as the median (min - max). The asterisks denote the significance levels: $* * * * p<0.0001$, $* * P<0.01$, $* * * p<0.001$, $* p<0.05$.

Abbreviations: JDNW, Jieduan-Niwan; ACLF, acute-on-chronic liver failure; TUNEL assay, terminal deoxynucleotidyl transferase dUTP nick end labeling assay; SD, standard deviation. 

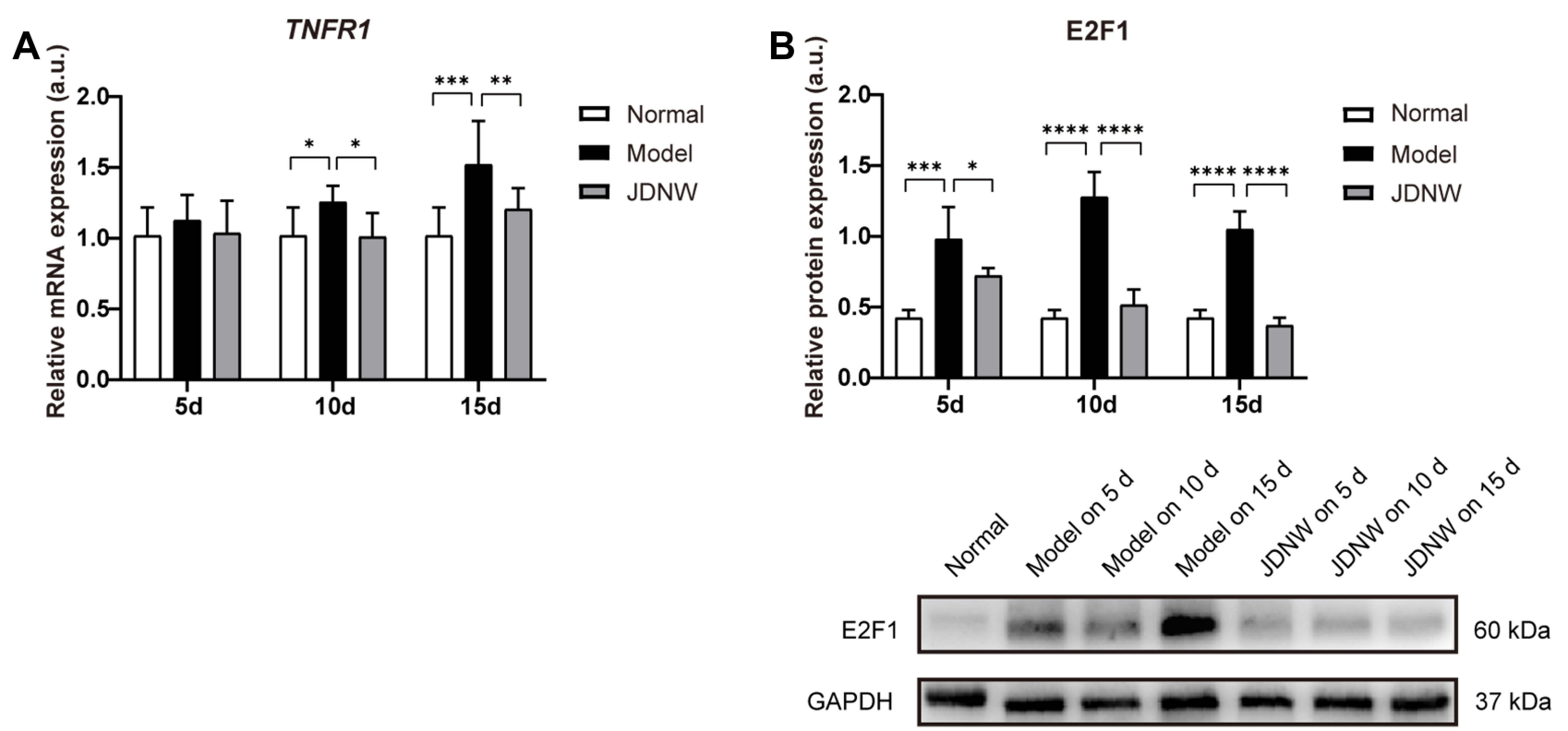

Figure 4 The JDNW formula inhibited the expression of TNFRI and E2FI in ACLF rats.

Notes: (A) Relative transcript levels of TNFRI in the different groups were detected by RT-qPCR. (B) The expression of E2FI in the different groups was detected by Western blot analysis. GAPDH was used as the loading control. The above results were expressed as mean \pm SD, asterisks denote the significance levels: $* P<0.05$, $* * * P<$ 0.001 , *** $<0.01$, ***** $<0.0001$

Abbreviations: JDNW, Jieduan-Niwan; TNFRI, tumor necrosis factor receptor I; E2FI, E2F transcription factor I; ACLF, acute-on-chronic liver failure; RT-qPCR, quantitative real-time PCR; SD, standard deviation.

\section{The JDNW Formula Inhibited}

\section{E2FI-Mediated Hepatocyte Apoptosis via the p53-Dependent Apoptosis Pathway in ACLF Rats}

To explore whether the JDNW formula could inhibit E2F1-mediated hepatocyte apoptosis via the p53-dependent apoptosis pathway and protect hepatocytes in ACLF rats, the expression of the transcripts of $p 53$ and $p 14^{A R F}$ in this pathway was analyzed by RT-qPCR (Figure 5A). The results showed that, compared with the normal group, the expression of the transcript levels of $p 53$ and $p 14^{A R F}$ was significantly enhanced at all time points in the model groups, with a gradually increasing trend over the entire time period (each $P<0.0001$ ). Interestingly, the expression of the transcripts of $p 53$ and $p 14^{A R F}$ decreased significantly after JDNW treatment $(p 53: P=0.0073, P<$ $0.0001, P<0.0001 ; p 14^{A R F}: P=0.0660, P<0.0001, P<$ $0.0001)$. In addition, the expression of the initiator caspase Caspase- 9 and its active form cleaved Caspase-9, as well as the executioner caspases Caspase-3/-6/-7 and their active forms cleaved Caspase-3/-6/-7, was detected by Western blot. The activity of Caspase was calculated by the ratio of cleaved Caspase/(Caspase+cleaved Caspase) (Figure 5B). The results showed that, compared with the normal group, the expression of cleaved Caspase-9/-3/-7/ (Caspase-9/-3/-7+cleaved Caspase-9/-3/-7) increased in the model groups on the 5th, 10th and 15th $\mathrm{d}$ (cleaved Caspase-9/(Caspase-9+cleaved Caspase-9): $P=0.0012$, $P=0.0001, P=0.0017$; cleaved Caspase-3/(Caspase-3 +cleaved Caspase-3): each $P<0.0001$; cleaved Caspase-7/ (Caspase-7+cleaved Caspase-7): $P=0.0067, P=0.0002$, $P<0.0001$ ), but decreased significantly in the JDNW groups. Specifically, for the JDNW groups, the downregulation of cleaved Caspase-9/(Caspase-9+cleaved Caspase-9) observed on the 5th $\mathrm{d}(P=0.0059)$, the significant down-regulation of cleaved Caspase-3/(Caspase-3 +cleaved Caspase-3) was noted on the 5th, 10th and 15th $\mathrm{d}(P=0.0009, P=0.0001, P<0.0001)$, and the significant decrease in cleaved Caspase-7/(Caspase-7+cleaved Caspase-7) was seen on both the 10th and 15th $\mathrm{d}(P=$ $0.0178, P<0.0001)$. Then, there was no statistically significant difference in the activity of Caspase- 6 among the groups over the entire time period. Hence, the data indicated that the JDNW formula reduced the hepatocyte apoptosis of ACLF rats by inhibiting the core genes, $p 53$ and $p 14^{A R F}$, and proteins, cleaved Caspase-9/-3/-7 but not cleaved Caspase-6, in E2F1-mediated p53-dependent apoptosis pathway. 


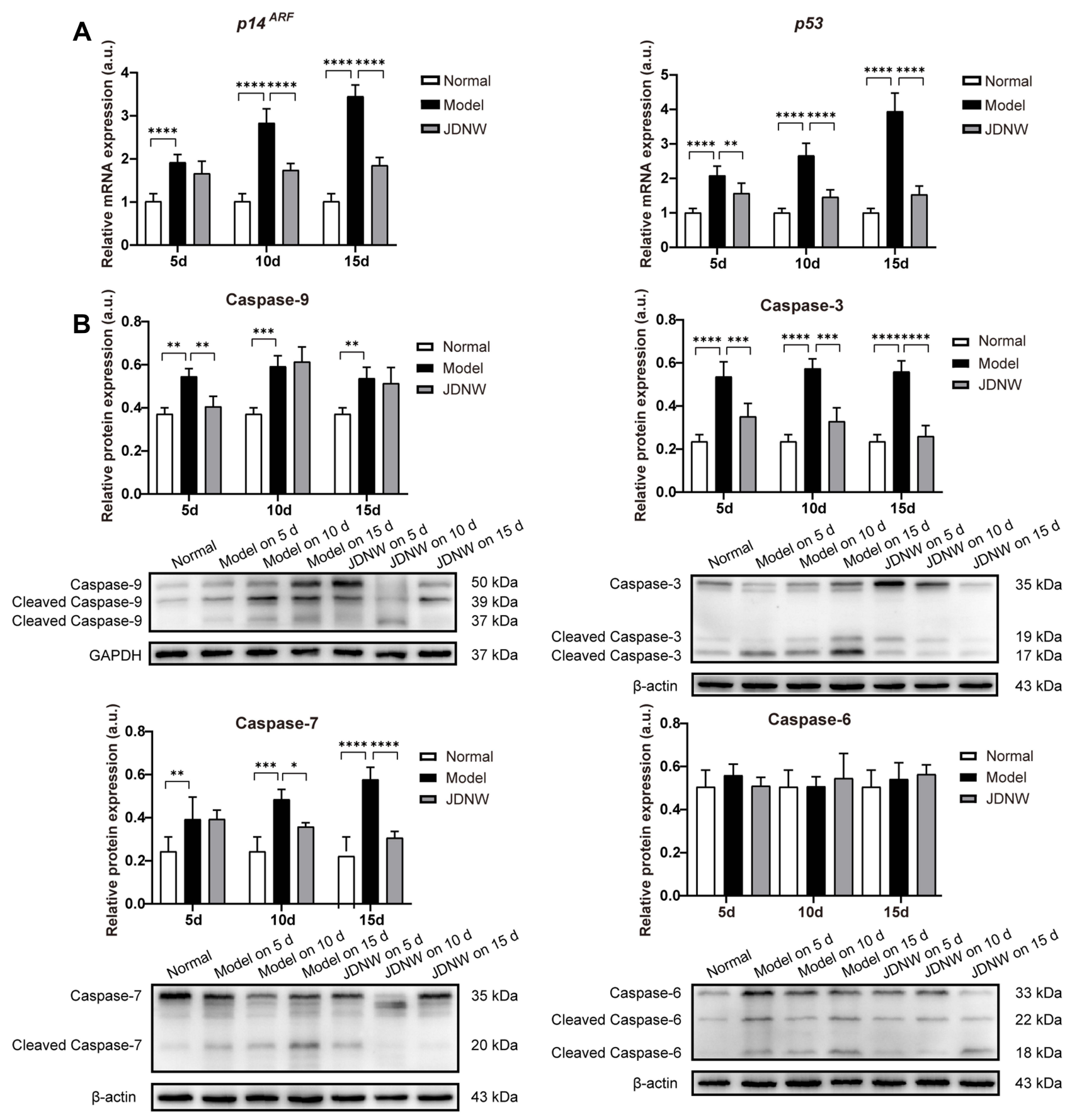

Figure 5 The JDNW formula inhibited E2FI-mediated hepatocyte apoptosis via the p53-dependent apoptosis pathway in ACLF rats.

Notes: (A) Relative transcript levels of $p / 4^{A R F}$ and $p 53$ in the different groups were detected by RT-qPCR. (B) The expression of caspases Caspase-9/-3/-6/-7 and their active forms cleaved Caspase-9/-3/-6/-7 in the different groups were detected by Western blot analysis. $\beta$-actin was used as the loading control of cleaved Caspase-3/-6/-7 and Caspase-3/-6/-7. GAPDH was used as the loading control of cleaved Caspase-9 and Caspase-9. The results of Caspase-6 were expressed as median (min - max), and the rest were expressed as mean $\pm S D$, asterisks denote the significance levels: $* * * * P<0.0001$, $* * P<0.01$, $* * * P<0.001, * P<0.05$.

Abbreviations: JDNW, Jieduan-Niwan; E2FI, E2F transcription factor I; $p 53$, tumor protein 53; ACLF, acute-on-chronic liver failure; pI4 $4^{\mathrm{ARF}}$, alternative reading frame product of the INK4A/ARF locus; RT-qPCR, quantitative real-time PCR; Caspases, cysteinyl aspartate-specific proteinase; SD, standard deviation. 

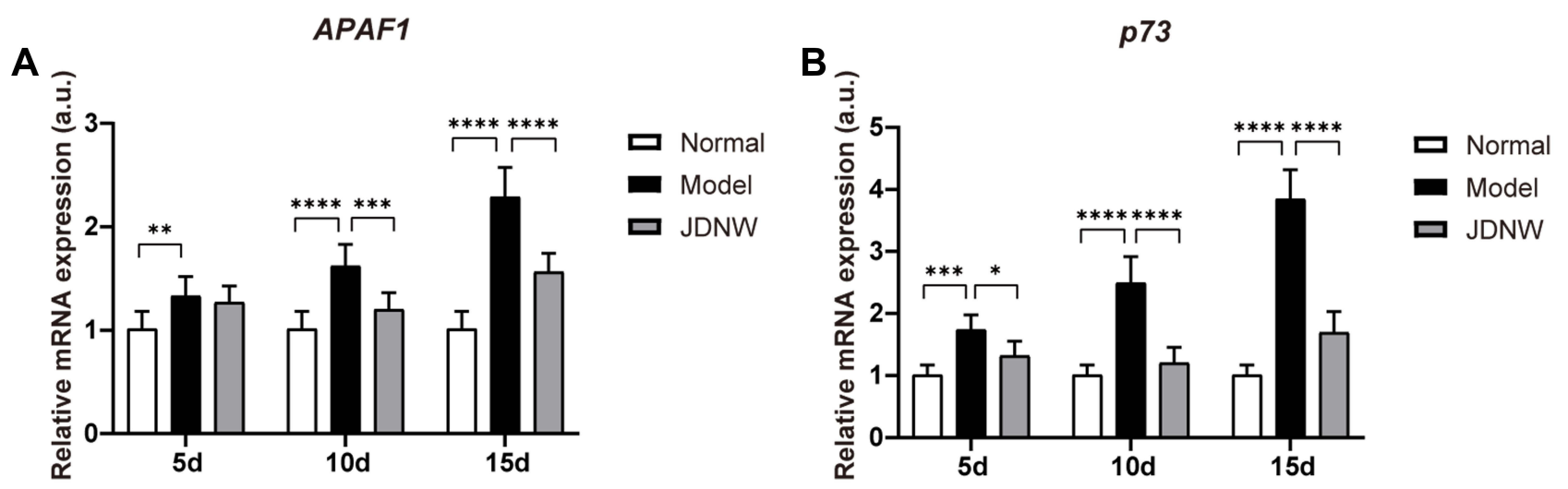

Figure 6 The JDNW formula inhibited E2FI-mediated hepatocyte apoptosis via the non-p53- dependent apoptosis pathways in ACLF rats.

Notes: (A) Relative transcript levels of APAFI in the different groups were detected by RT-qPCR. (B) Relative transcript levels of $p 73$ in the different groups were detected by RT-qPCR. The above results were expressed as mean \pm SD, asterisks denote the significance levels: $* * P<0.01, * * * * P<0.000 \mathrm{I}, * * * P<0.00 \mathrm{I}$, $* P<0.05$.

Abbreviations: JDNW, Jieduan-Niwan; E2FI, E2F transcription factor I; p53, tumor protein 53; ACLF, acute-on-chronic liver failure; APAFI, apoptotic peptidase activating factor I; RT-qPCR, quantitative real-time PCR; p73, tumor protein 73, the homologue of p53; SD, standard deviation.

\section{The JDNW Formula Inhibited}

\section{E2FI-Mediated Hepatocyte Apoptosis via} the Non-P53-Dependent Apoptosis

\section{Pathways in ACLF Rats}

To determine whether the JDNW formula could inhibit E2F1-mediated hepatocyte apoptosis via the non-p53dependent apoptosis pathways and protect hepatocytes in ACLF rats, the expression of the transcripts of two vital genes, $A P A F 1$ and $p 73$, from the pathways was analyzed by RT-qPCR (Figure 6A and B). The results showed that, compared with the normal group, the expression of the transcript levels of $A P A F 1$ and $p 73$ increased significantly in the model groups of ACLF rats on the 5th, 10th and 15th d (APAF1: $P=0.0084, P<0.0001, P<0.0001 ; p 73$ : $P=0.0004, P<0.0001, P<0.0001)$. These effects were notably inhibited by JDNW treatment, as seen by the significant decrease in $A P A F 1$ and $p 73$ in the JDNW groups compared with the model groups (APAF1: $P=$ 0.5896, $P=0.0008, P<0.0001 ; p 73: P=0.0309, P<$ $0.0001, P<0.0001)$. Therefore, together with the results of the expression of caspase proteins (Figure 5B), the data suggested that the JDNW formula inhibited the activation of the non-p53-dependent apoptosis pathways, induced by the overexpression of E2F1 in ACLF model rats, via the down-regulation of the expression of the two key genes, $A P A F 1$ and $p 73$, and caspase proteins, cleaved Caspase-9/$3 /-7$, but not cleaved Caspase- 6 .

\section{The JDNW Formula Inhibited}

E2FI-Mediated Hepatocyte Apoptosis via the Blocking Anti-Apoptotic Signaling

\section{Pathways in ACLF Rats}

To confirm whether the JDNW formula could inhibit the E2F1-mediated apoptosis of hepatocytes in ACLF rats via the blocking anti-apoptotic signaling pathways, the expression of the transcripts of TRAF2 (Figure 7A), Bcl-2 (Figure 7B), and $\mathrm{Mcl}-1$ (Figure 7C), which are the key factors in the pathways, was detected by RTqPCR. The data showed that, compared with the normal group, the expression of the transcripts of TRAF2, Mcl-1, and $B c l-2$ decreased significantly at each time point in the model groups (TRAF2: each $P<0.0001$; Mcl-1: each $P<0.0001 ;$ Bcl-2: $P=0.5884, P=$ $0.0148, P=0.0005)$. The transcript levels of TRAF2, Mcl-1, and Bcl-2 increased significantly in the JDNW groups, with the notable increase in TRAF2 and $\mathrm{Mcl}-1$ seen on the 15 th $\mathrm{d}$ (each $P<0.0001$ ) and the significant increase in $\mathrm{Bcl}-2$ observed on both the 10th and 15th $\mathrm{d}(P=0.0401, P<0.0001)$. These findings indicated that the JDNW formula inhibited hepatocyte apoptosis through the up-regulation of the expression of TRAF2, Mcl-1, and Bcl-2 and the down-regulation of cleaved Caspase-9/-3/-7 but not cleaved Caspase- 6 (Figure 5B) from the blocking anti-apoptotic signaling pathways. 

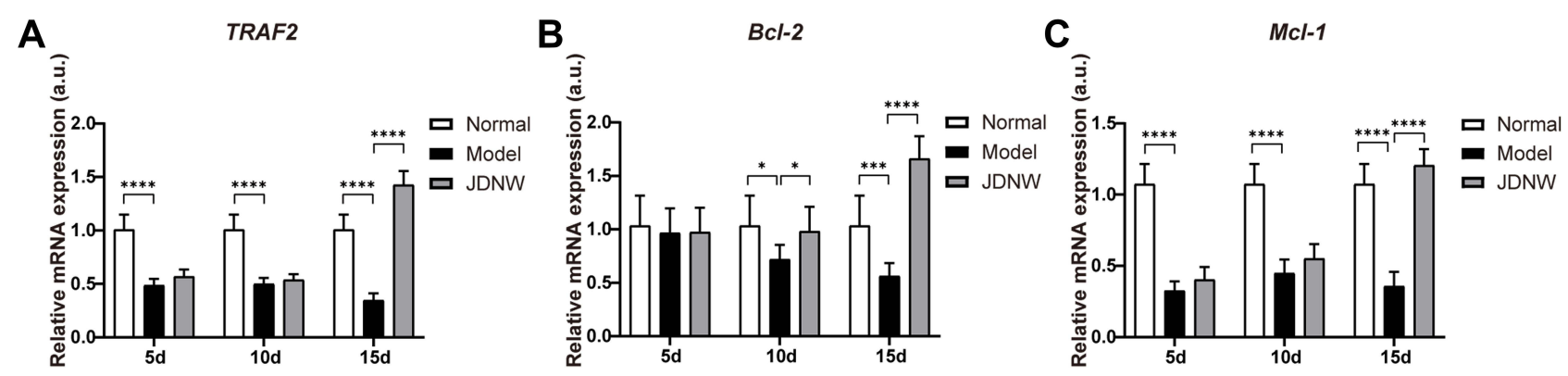

Figure 7 The JDNW formula inhibited E2FI-mediated hepatocyte apoptosis via the blocking anti-apoptotic signaling pathways in ACLF rats.

Notes: (A) Relative transcript levels of TRAF2 in the different groups were detected by RT-qPCR. (B) Relative transcript levels of Bcl-2 in the different groups were detected by RT-qPCR. (C) Relative transcript levels of $\mathrm{Mcl}-\mathrm{I}$ in the different groups were detected by RT-qPCR. The above results were expressed as mean \pm SD, asterisks denote the significance levels: $* * * * P<0.000 \mathrm{I}, * P<0.05$, $* * * P<0.001$.

Abbreviations: JDNW, Jieduan-Niwan; E2FI, E2F transcription factor I; ACLF, acute-on-chronic liver failure; TRAF2, TNF receptor associated factor 2; RT-qPCR, quantitative real-time PCR; Bcl-2, B-cell lymphoma 2; Mcl-I, myeloid cell leukemia I; SD, standard deviation.

\section{Discussion}

In recent years, clinical studies have shown that the JDNW formula is a promising prescription for the treatment of ACLF, which could improve the liver function and other symptoms and reduce mortality in patients. ${ }^{33}$ In the present study, the main constituents of the JDNW formula and their concentrations were revealed by HPLC analysis, including catalpol $(0.1324 \mathrm{mg} / \mathrm{mL})$, GA $(0.0902 \mathrm{mg} / \mathrm{mL})$, 3,4-dihydroxybenzaldehyde $(0.0073 \mathrm{mg} / \mathrm{mL}), \quad$ CA $(0.0266 \mathrm{mg} / \mathrm{mL}), \quad$ NG-R1 $\quad(0.1905 \mathrm{mg} / \mathrm{mL}), \quad$ Sal B $(0.5436 \mathrm{mg} / \mathrm{mL})$, and GRb1 $(0.1834 \mathrm{mg} / \mathrm{mL})$. Sal $\mathrm{B}$ and 3,4-dihydroxybenzaldehyde are polyphenolic compounds in Salvia miltiorrhiza Bunge; NG-R1 and GRb1 are the predominant active ingredients of Panax notoginseng (Burkill) F. H. Chen; Catalpol is an iridoid glycoside abundant in the roots of Rehmannia glutinosa (Gaertn.) DC; GA is a natural polyphenolic in Lysimachia christinae Hance and Viscum coloratum (Kom.) Nakai; and CA is one of the vital components of Astragalus membranaceus (Fisch.) Bunge. These ingredients possess anti-oxidation, anti-inflammatory, anti-apoptosis and other pharmacological properties, and play important roles in the treatment of fatty liver, acute liver injury, liver failure and other diseases. Here Sal B was the most abundant ingredient in JDNW formula. It has been reported that Sal B could significantly scavenge free radicals, maintain mitochondrial membrane permeability, ensure basic energy metabolism, and inhibit the activation of apoptosis-related factors such as Caspase-3 and Bcl-2 by oxidative stress. ${ }^{41}$ Moreover, Sal B reduces inflammation and hepatocyte apoptosis by activating the SIRT1/PGC-1 $\alpha$ signaling pathway to attenuate liver damage caused by sepsis. ${ }^{42}$ In addition, Sal B is recognized to prominently alleviate liver fibrosis in chronic hepatitis B virus (HBV) patients without causing obvious side effects. ${ }^{41}$ Furthermore, Sal $B$ protects the integrity of the lysosomal membrane by upregulating the expression of LAMP1, thereby reducing the leakage of $\mathrm{CatB} / \mathrm{D}$ into the cytosol and protecting hepatocytes from death. ${ }^{43}$ NG-R1 is capable of reducing intestinal ischemia-reperfusion injury induced microvascular hyperpermeability, the production of inflammatory factors, improving intestinal and liver microcirculation, and inhibiting epithelial cell apoptosis. ${ }^{44}$ GRb1 alleviates hepatocytic apoptosis via peroxisome proliferator-activated receptor $\gamma .{ }^{45}$ Catalpol improves the mitochondrial membrane potential and regulates inflammatory cytokines and the expression of apoptotic proteins through antioxidant effects and protects the liver from cholestasis-mediated injury. ${ }^{46}$ GA inhibits lipid accumulation, apoptosis and inflammation in hepatocytes by activating AMPK. ${ }^{47} \mathrm{CA}$ alleviates oxidative stress in the liver by scavenging mitochondria and intracellular ROS, inhibits steatosis, hepatocyte apoptosis and liver fibrosis, and improves alcoholic liver injury. ${ }^{48}$ 3,4-dihydroxybenzaldehyde induces S-Phase arrest and apoptosis by stimulating the p $27^{\mathrm{KIP} 1}$-Cyclin A/ D1-CDK2 and mitochondrial apoptotic pathways in HT-29 cells. ${ }^{49}$ In conclusion, these findings suggest that the 7 components in JDNW formula play the anti-apoptotic and hepatoprotective role. It has been reported recently that the JDNW method has been utilized to treat patients with the novel coronavirus disease 2019 (COVID-19) severe novel pneumonia, with remarkable outcomes, at Beijing Ditan Hospital, which is affiliated to the Capital Medical University, a designated institution treating the novel COVID-19 in China. 
ACLF is a syndrome that severe decompensation in liver function and massive or submassive necrosis of liver parenchyma caused by acute injury on the background of chronic liver disease. Liver cirrhosis, induced by the immunologic stimulant HSA, is very similar to the pathological basis of ACLF. D-GalN and LPS could cause macrophages to produce more inflammatory factors, which induce severe liver damage accompanied by apoptosis, liver necrosis, and other organ damage symptoms, ultimately leading to acute liver failure (ALF). ${ }^{50} \mathrm{D}-\mathrm{GalN} /$ LPS-induced ALF is similar to the acute injury process of ACLF. The ACLF rat model simulates part of the pathophysiological process of ALF based on the chronic liver disease at the animal level. In the present study, we found that the PTA of rats in the model groups decreased significantly, suggesting that the ACLF rats had coagulation dysfunction, which is consistent with the clinical manifestations of ACLF patients. The results of HE staining and reticular fiber staining showed that the pathological phenomena such as edema and deformation of liver cells, accompanied by inflammatory cell infiltration, prominent liver fibrosis, widespread formation of pseudolobules, and reticular fibers were thick and numerous in the portal area; massive and submassive necrosis of liver tissues were also clearly observed. Over time, the degree of the pathological changes in the ACLF became increasingly severe. The results of TUNEL assay and flow cytometry showed that the hepatocyte apoptosis index and apoptosis rate increased significantly. Moreover, the previous studies revealed that the levels of alanine transaminase (ALT), aspartate transaminase (AST), and total bilirubin (TBIL) increased in ACLF rats. ${ }^{8}$ With the electron microscope, it was observed that the liver cells were swollen, the nuclear abnormality was serious, the rough endoplasmic reticulum was expanded and broken, the mitochondrial membrane was ruptured, cristae had disappeared, and vacuolization and apoptotic bodies significantly increased. ${ }^{34}$ These results indicated that the ACLF model was established successfully. After the treatment with the JDNW formula, the pathological damage was significantly alleviated, the hepatocyte apoptosis rate and apoptosis index significantly decreased, and the liver function and coagulation function improved in ACLF rats. These results suggest that the JDNW formula could effectively inhibit the excessive apoptosis of hepatocyte, decrease the level of hepatic tissue damage and necrosis, and protect the liver of ACLF rats.
Although the precise definition of ACLF remains controversial, with a consensus yet to be reached on the matter between Eastern and Western researchers, it is generally believed that the host immune response and the inflammatory cascade characterize the fundamental pathological mechanisms of ACLF. ${ }^{51-53}$ When D-GalN and LPS reach the liver, the Kupffer cells and blood sinusoidal endothelial cells of the liver are stimulated to produce and release a large number of inflammatory factors, particularly TNF$\alpha$ and IL-6, which could promote the proliferation and differentiation of cytotoxic $\mathrm{T}$ lymphocytes, enhance the cytolytic function of Natural Killer (NK) cells against physiologically stressed cells, provoke cellular immunity, and cause different degrees of inflammatory response and immune damage. ${ }^{54-57}$ TNF- $\alpha$ binds to TNFR1 forms an oligomer, which could transmit the signals activated by the extracellular TNF- $\alpha$ to the cell, regulates tyrosine phosphorylation and the expression of E2F1 through the signal transducer and activator of transcription 3 (STAT3), ${ }^{9}$ and then produces a series of molecular events. Our previous experimental studies found that the serum and liver tissues of ACLF rats showed increased levels of TNF- $\alpha$ and IL-6; the opposite effects were observed in the JDNW formula groups, with the most significant difference found mainly on the 15 th day. ${ }^{34}$ In the present study, the data showed that, compared with the normal group, the expression of TNFR1 and E2F1 in the hepatic tissues of rats on the 5th, 10th, and 15th $d$ in the model groups increased significantly. Compared with the model groups, the expression of the factors TNFR1 and E2F1 decreased highly in the JDNW groups at each corresponding time point, with the most significant down-regulation of TNFRl transcripts observed on the 10th and 15th $\mathrm{d}$ and the most significant decrease in E2F1 seen on the 5th, 10th and 15th d. All data suggested that the expressions of TNFR1 and E2F1 could be effectively down-regulated after treatment with the JDNW formula, which might have contributed to the protection of the hepatic cells of ACLF rats by reducing TNF$\alpha$ and IL-6 release and the expression of transcripts of TNFR1, thus reducing the combination of TNF- $\alpha$ and TNFR1 and the expression of E2F1.

The pathogenic mechanisms of ACLF are closely related to the excessive apoptosis of hepatic cells. ${ }^{5,6,8}$ E2F1-mediated apoptosis pathways, which are among the most important apoptotic pathways, ${ }^{58,59}$ play an important role in ACLF caused by excessive apoptosis of liver cells. ${ }^{8}$ The increased expression of E2F1 could enhance the transcripts of $p 14^{A R F}$ and thus enhance the expression level of 
$p 53$ to initiate apoptosis. ${ }^{10,12-14}$ The present study showed that the expression of the transcript levels of $p 53$ and $p 14^{A R F}$ in the model groups increased significantly on the 5th, 10th, and 15th d, with gradual increase over the entire time period. The expression of $p 53$ and $p 14^{A R F}$ decreased significantly after treatment with JDNW, with the most significant decrease in both genes observed on the 10th and 15th d. APAF1 is a direct transcriptional target of $p 53,{ }^{15-17}$ the up-regulation of the expression of p53 could increase the expression of APAF1, which activates Caspase- 9 into active form cleaved Caspase-9, then the activated Caspase- 9 cleaves and activates Caspase- 3 to initiate downstream caspase-cascade system. ${ }^{10,17-19}$ In the present study, compared with the normal group, the expression of the transcript of $A P A F 1$ and the activities of Caspase- $9 /-3 /-7$ increased significantly in the model groups of ACLF rats on the 5th, 10th and 15th d. These effects were notably inhibited by JDNW treatment. Specifically, the most significant down-regulation of the expression of the transcripts of APAF1 in the JDNW groups was presented on the 10th and 15th d, the downregulation of the activity of Caspase-9 noted on the 5th $d$, Caspase- 3 activity decreased significantly on the 5th, 10th and 15 th $\mathrm{d}$, and the significant decrease in the activity of Caspase- 7 seen on both the 10th and 15th $\mathrm{d}$. There were no significant changes in the activity of Caspase-6, which suggested that Caspase- 6 may not be recruited in the caspase-cascade system here. The data suggest that the JDNW formula can inhibit E2F1-mediated hepatocyte apoptosis via the p53-dependent apoptosis pathway in ACLF rats and can also have a protective effect on hepatocytes. In the absence of p53, E2F1 can directly activate several apoptosis-induced genes, such as $p 73 .^{10,15,17,23}$ Besides, E2F1 does not rely on the release of cytochrome $c$ in the mitochondria. The alternative pathway, which depends on increasing the expression of APAF 1 , is directly induced by E2F1 to activate Caspase-9 without the accumulation of cytochrome $c,{ }^{17}$ and this process is concomitant with the initiation of a caspase-cascade for apoptosis via the non-p53-dependent pathways. The results showed that, compared with the normal group, the expression of the transcripts of $p 73$ increased significantly in the model groups of ACLF rats on the 5th, 10th and 15th d. These effects were notably inhibited by JDNW treatment. Hence, these results suggest that the JDNW formula can alleviate hepatocyte apoptosis of ACLF rats by modulating E2F1mediated, non-p53-dependent apoptosis pathways. Furthermore, E2F1 sensitizes cells to apoptosis-promoting stimuli and induces apoptosis through the NF- $\mathrm{BB}$ signaling pathway. The overexpression of E2F1 can down-regulate the expression level of the signal transduction molecule TRAF2, mediate signal transmission to the downstream, inhibit NF- $\mathrm{KB},{ }^{60}$ and block anti-apoptotic signals. In addition, it inhibits the expression of Mcl-1 and Bcl-2, which could change the mitochondrial permeability and promote the release of apoptotic forming factors such as cytochrome $c$ from the mitochondria and induce apoptosis. ${ }^{61}$ The present results revealed that the transcripts expression of TRAF2, Mcl-1 and Bcl-2 decreased significantly at each time point in the model groups compared with the normal group. But in the JDNW groups the transcript levels of TRAF2, $\mathrm{Mcl}-1$ and $\mathrm{Bcl}-2$ were highly up-regulated, with the notable increase in TRAF2 and Mcl-1 seen on the 15th $\mathrm{d}$ and the significant increase in $\mathrm{Bcl}-2$ observed on both the 10 th and 15th $\mathrm{d}$. These findings indicated that the JDNW formula could alleviate hepatocyte apoptosis of ACLF rats by regulating the E2F1-mediated blocking anti-apoptotic signaling pathways. The schematic diagram of JDNW's regulation of E2F1-mediated apoptosis pathways in hepatocyte is shown in Figure 8. To sum up, the data suggest that the JDNW formula can effectively inhibit the excessive apoptosis and protect the liver cells of ACLF rats by modulating the three E2F1-mediated apoptosis pathways. Appropriate inhibition of the E2F1-mediated hepatocyte apoptosis pathways is a potential therapeutic target of ACLF.

Taken together, the present study provides a better understanding of the histopathological and molecular mechanisms of the JDNW formula in the treatment of ACLF rats. The JDNW formula can act as a liver protectant and participate in the modulation of the three E2F1mediated apoptosis pathways.

\section{Conclusions}

In summary, the present study has shown that the JDNW formula could ameliorate coagulation function and pathological damage to the liver, decrease the hepatocyte apoptosis index and apoptosis rate, and inhibit the excessive apoptosis of liver cells by regulating the E2F1-mediated hepatocyte apoptosis pathways in ACLF rats, with the most significant effects observed mainly on the 15th d. These findings indicate that the E2F1-mediated apoptotic pathways play an important role in ACLF progression, suggesting these avenues as a potential and worthy therapeutic target of ACLF treatment. It is hoped that this study has laid the foundation for future research on the 


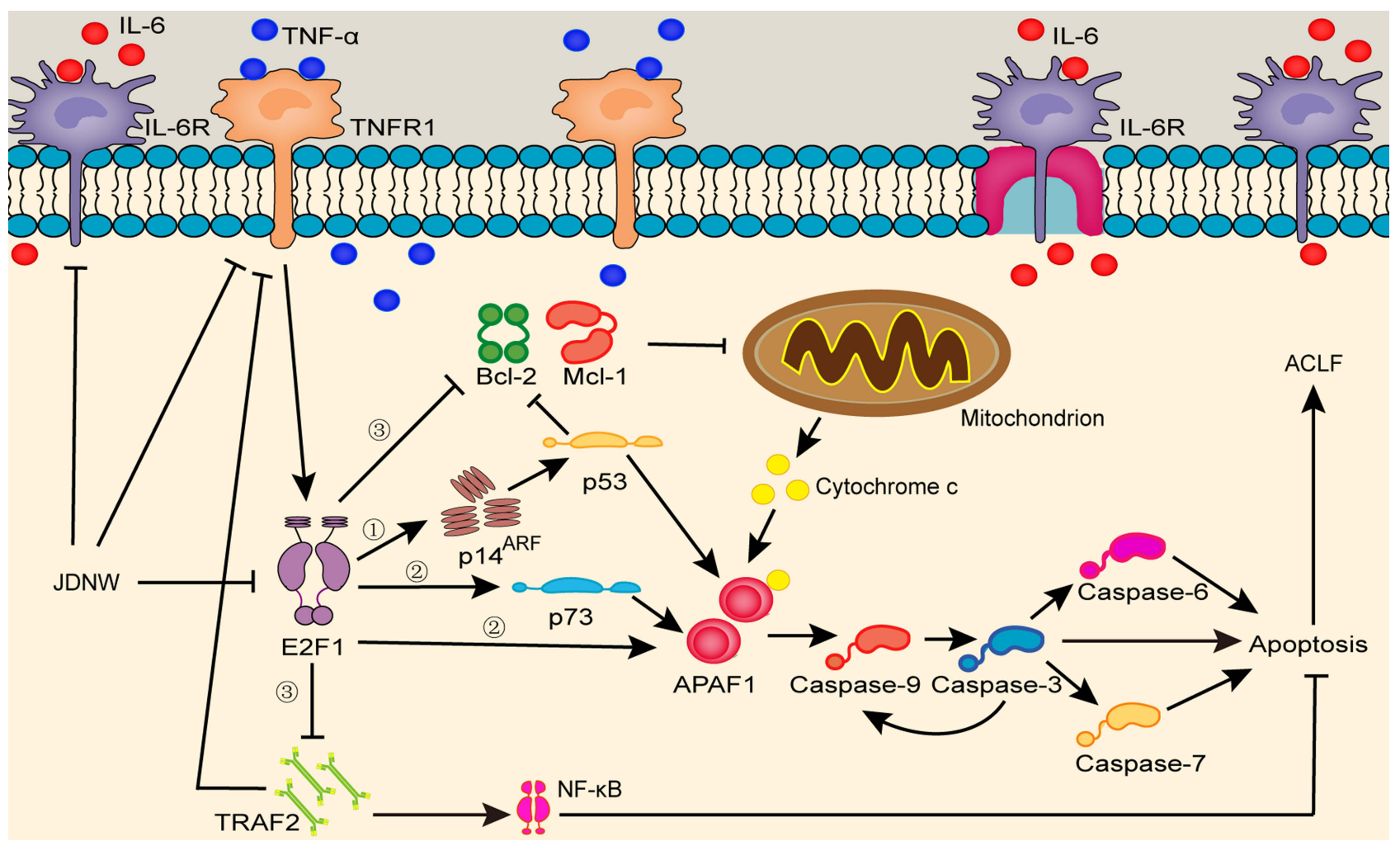

Figure 8 JDNW's regulation of E2FI-mediated apoptosis pathways in hepatocyte.

Notes: (1) The p53-dependent apoptosis pathway. (2) The non-p53-dependent apoptotic pathways. (3) The blocking anti-apoptotic signal pathways.

Abbreviations: JDNW, Jieduan-Niwan; TNF- $\alpha$, tumor necrosis factor- $\alpha$; TNFRI, tumor necrosis factor receptor I; IL-6, interleukin-6; IL-6R, interleukin-6 receptor; E2FI, E2F transcription factor I; pl4 ${ }^{A R F}$, alternative reading frame product of the INK4A/ARF locus; p53, tumor protein 53; APAFI, apoptotic peptidase activating factor I; Caspases, cysteinyl aspartate-specific proteinase; p73, tumor protein 73, the homologue of p53; TRAF2, TNF receptor associated factor 2; Bcl-2, B-cell lymphoma 2; Mcl-I, myeloid cell leukemia I; NF-kB, nuclear factor kappa-light-chain-enhancer of activated B cells.

role of the JDNW formula in the management of ACLF and will provide a better time window for such research.

\section{Ethical Approval}

All animal experiments were approved by the Animal Ethics Committee of the Capital Medical University (approval ID: AEEI-2015-187) and performed in accordance with the Guide for the Care and Use of Laboratory Animals of the Beijing Municipal Government.

\section{Acknowledgments}

This work was supported by the National Natural Science Foundation of China [grant number 81573767], and the National Key Research and Development Program of China - Research on Inheritance and Innovation of Experience (Integration of Tao and Shu) of Illustrious Senior Traditional Chinese Medicine Practitioners by adopting Multiple Research Methods [grant number 2018YFC1704100] and its subproject-Research on the Academic Viewpoints, Unique Diagnostic and Treatment
Methods and Major Diseases Prevention and Treatment Experience of Illustrious Senior Traditional Chinese Medicine Practitioners in Eastern China [grant number 2018YFC1704102]. We gratefully acknowledge the contributions of Dr Weina Hou (Department of Biology, University of Minho, Portugal) for providing language assistance, including writing assistance, organizing, reading, correcting and vetting the manuscript.

\section{Disclosure}

The authors report no conflicts of interest in relation to this work.

\section{References}

1. Liver Failure and Artificial Liver Group, Chinese Society of Infectious Diseases, Chinese Medical Association, Severe Liver Disease and Artificial Liver Group, Chinese Society of Hepatology, Chinese Medical Association. [Guideline for diagnosis and treatment of liver failure (2018)]. Chin J Clin Hepatol. 2019;35(1):38-44. Chinese. doi:10.3969/j.issn.1001-5256 
2. Trebicka J, Sundaram V, Moreau R, Jalan R, Arroyo V. Liver transplantation for acute-on-chronic liver failure: science or fiction? Liver Transpl. 2020;26(7):906-915. doi:10.1002/1t.25788

3. Trebicka J, Gu W, Ibáñez-Samaniego L, et al. Rebleeding and mortality risk are increased by ACLF but reduced by pre-emptive TIPS $J$ Hepatol. 2020;73(5):1082-1091. S0168-8278(20)30236-1. doi:10.1016/j.jhep.2020.04.024

4. Singal AK, Ahmed Z, Axley P, et al. Hospitalizations for acute on chronic liver failure at academic compared to non-academic centers have higher mortality. Dig Dis Sci. 2021;66(4):1306-1314. doi:10.1007/s10620-020-06263-w

5. Cai J, Han T, Nie C, et al. Biomarkers of oxidation stress, inflammation, necrosis and apoptosis are associated with hepatitis B-related acute-on-chronic liver failure. Clin Res Hepatol Gastroenterol. 2016;40(1):41-50. doi:10.1016/j.clinre.2015.06.009

6. Xue R, Yang J, Jia L, et al. Mitofusin2, as a protective target in the liver, controls the balance of apoptosis and autophagy in acute-onchronic liver failure. Front Pharmacol. 2019;10:601. doi:10.3389/ fphar.2019.00601

7. Wu Y, Ding J, Sun Q, et al. Long noncoding RNA hypoxia-inducible factor 1 alpha-antisense RNA 1 promotes tumor necrosis factor- $\alpha$ induced apoptosis through caspase 3 in Kupffer cells. Medicine. 2018;97(4):e9483. doi:10.1097/MD.0000000000009483

8. Yang W, Hao Y, Hou W, et al. Jieduan-Niwan formula reduces liver apoptosis in a rat model of acute-on-chronic liver failure by regulating the E2F1-mediated intrinsic apoptosis pathway. Evid Based Complement Alternat Med. 2019;2019:8108503. doi:10.1155/2019/8108503

9. Kubota K, Inoue K, Hashimoto $\mathrm{R}$, et al. Tumor necrosis factor receptor-associated protein 1 regulates cell adhesion and synaptic morphology via modulation of $\mathrm{N}$-cadherin expression. $J$ Neurochem. 2009;110(2):496-508. doi:10.1111/j.1471-4159.2009.06099.x

10. Pflaum J, Schlosser S, Müller M. p53 family and cellular stress responses in cancer. Front Oncol. 2014;4:285. doi:10.3389/ fonc. 2014.00285

11. Wu Z, Zheng S, Yu Q. The E2F family and the role of E2F1 in apoptosis. Int $J$ Biochem Cell Biol. 2009;41(12):2389-2397. doi:10.1016/j.biocel.2009.06.004

12. Phillips AC, Vousden KH. E2F-1 induced apoptosis. Apoptosis. 2001;6(3):173-182. doi:10.1023/A:1011332625740

13. Lv H, Liu R, Fu J, et al. Epithelial cell-derived periostin functions as a tumor suppressor in gastric cancer through stabilizing p53 and E-cadherin proteins via the $\mathrm{Rb} / \mathrm{E} 2 \mathrm{~F} 1 / \mathrm{p} 14 \mathrm{ARF} / \mathrm{Mdm} 2$ signaling pathway. Cell Cycle. 2014;13(18):2962-2974. doi:10.4161/ 15384101.2014.947203

14. Moon NS, Di Stefano L, Morris EJ, Patel R, White K, Dyson NJ. $\mathrm{E} 2 \mathrm{~F}$ and $\mathrm{p} 53$ induce apoptosis independently during drosophila development but intersect in the context of DNA damage. PLoS Genet. 2008;4(8):e1000153. doi:10.1371/journal.pgen.1000153

15. Ginsberg D. E2F1 pathways to apoptosis. FEBS Lett. 2002;529 (1):122-125. doi:10.1016/s0014-5793(02)03270-2

16. Moroni MC, Hickman ES, Lazzerini Denchi E, et al. Apaf-1 is a transcriptional target for E2F and p53. Nat Cell Biol. 2001;3 (6):552-558. doi:10.1038/35078527

17. Furukawa Y, Nishimura N, Furukawa Y, et al. Apaf-1 is a mediator of E2F-1-induced apoptosis. J Biol Chem. 2002;277(42):39760-39768. doi:10.1074/jbc.M200805200

18. Phillips AC, Ernst MK, Bates S, Rice NR, Vousden KH. E2F-1 potentiates cell death by blocking antiapoptotic signaling pathways. Mol Cell. 1999;4(5):771-781. doi:10.1016/s1097-2765(00)80387-1

19. Fan T, Han L, Cong R, Liang J, Zhang Y-S. Caspase family proteases and apoptosis. Acta Biochim Biophys Sin. 2005;37(11):719-727. doi:10.1111/j.1745-7270.2005.00108.x

20. Wang X, Cao Q, Zhang Y, Su X. Activation and regulation of Caspase- 6 and its role in neurodegenerative diseases. Annu Rev Pharmacol Toxicol. 2015;55(1):553-572. doi:10.1146/annurevpharmtox-010814-124414
21. Islam MS, Takano R, Yokochi T, et al. Programmed expression of pro-apoptotic BMCC1 during apoptosis, triggered by DNA damage in neuroblastoma cells. BMC Cancer. 2019;19(1):542. doi:10.1186/ s12885-019-5772-4

22. Aubrey BJ, Kelly GL, Janic A, Herold MJ, Strasser A. How does p53 induce apoptosis and how does this relate to p53-mediated tumour suppression? Cell Death Differ. 2018;25(1):104-113. doi:10.1038/ cdd.2017.169

23. Irwin M, Marin MC, Phillips AC, et al. Role for the $\mathrm{p} 53$ homologue p73 in E2F-1-induced apoptosis. Nature. 2000;407(6804):645-648. doi: $10.1038 / 35036614$

24. Vince JE, Pantaki D, Feltham R, et al. TRAF2 must bind to cellular inhibitors of apoptosis for tumor necrosis factor (TNF) to efficiently activate NF- $\mathrm{NB}$ and to prevent TNF-induced apoptosis. J Biol Chem. 2009;284(51):35906-35915. doi:10.1074/jbc.M109.072256

25. Croxton R, Ma Y, Song L, Haura EB, Cress DW. Direct repression of the Mcl-1 promoter by E2F-1. Oncogene. 2002;21(9):1359-1369. doi:10.1038/sj.onc. 1205157

26. Chen Z, Huang L, Li M, Meng L, Ying S, Xu A. Inhibitory effects of isocryptotanshinone on gastric cancer. Sci Rep. 2018;8(1):9307. doi:10.1038/s41598-018-27638-0

27. Elkholi R, Floros KV, Chipuk JE. The role of BH3-only proteins in tumor cell development, signaling, and treatment. Genes Cancer. 2011;2(5):523-537. doi:10.1177/1947601911417177

28. Knezevic D, Brash DE. Role of E2F1 in apoptosis: a case study in feedback loops. Cell Cycle. 2004;3(6):729-732. doi:10.4161/ cc.3.6.907

29. Hernaez R, Solà E, Moreau R, Ginès P. Acute-on-chronic liver failure: an update. Gut. 2017;66(3):541-553. doi:10.1136/gutjnl-2016312670

30. China Association of Chinese Medicine. [Guide lines for clinical diagnosis and treatment of acute-on-chronic liver failure in traditional Chinese medicine]. J Clin Hepatol. 2019;35(03):494-503. Chinese. doi:10.3969/j.issn.1001-5256.2019.03.009

31. Qian Y. [Using Jieduan-Niwan method treats chronic severe hepatitis]. Beijing J Tradit Chin Med. 2008;27(02):85-87. Chinese. doi:10.16025/j.1674-1307

32. Li X. [Professor Qian ying's considerations and strategies on treatment of chronic severe hepatitis by blocking and reversing therapy]. Shanghai J Tradit Chin Med. 2007;41(1):1-4. Chinese. doi:10.16305/ j.1007-1334.2007.01.001

33. Hu J, Qian Y, Yao N, et al. [Treatment of chronic severe hepatitis B by Jieduan Niwan method]. Chin J Integr Tradit West Med Liver Dis. 2010;20(4):200-203. Chinese. doi:10.3969/j.issn.1005-0264

34. Hou W, Hao Y, Yang W, et al. [Effects of Jieduan Niwan prescription on IL- 6 and TNF- $\alpha$ in serum and hepatic tissue of acute-on-chronic liver failure rats]. Chin J Inf Tradit Chin Med. 2018;25(07):49-52. Chinese. doi:10.3969/j.issn.1005-5304.2018.07.012

35. Hao Y, Yang W, Hou W, et al. [Effects of Jieduan Niwan formula on the pathway of cell proliferation in ACLF rats]. Inf Tradit Chin Med. 2018;35(03):12-17. Chinese. doi:10.19656/j.cnki.1002-2406.180074

36. Hao Y, Yang W, Hou W, et al. [Regulation mechanism of Jieduan Niwan formula on E2F1 signaling pathway in rats with acute-on chronic hepatic failure]. Global Tradit Chin Med. 2018;11 (03):321-326. Chinese. doi:10.3969/j.issn.1674-1749

37. Liu Z, Wu W, Zhang Q. [The influence of the prescription of 'truncation and inverse draft' on the ET and TNF- $\alpha$ of acute-on-chronic liver failure model rats]. Chin J Med Guide. 2011;13(04):650-652. Chinese. doi:10.3969/j.issn.1009-0959.2011.04.054

38. Wang B, Wang Z, Yin W, Huang S, Li J. [Studies on experimental immune hepatic fibrosis model]. Natl Med J Chin. 1989;69 (09):503-505. Chinese.

39. Liu X, Meng Y, Chen Y, Zhang L, Duan Z. [Exploration of establishing rat model of ACLF in human serum album in-induced cirrhosis]. Chin J Gastroenterol Hepatol. 2008;17(10):790-793. Chinese. doi:10.3969/j.issn.1006-5709.2008.10.004 
40. Schindelin J, Arganda-Carreras I, Frise E, et al. Fiji: an open-source platform for biological-image analysis. Nat Methods. 2012;9 (7):676-682. doi:10.1038/nmeth.2019

41. Gong Y, Li D, Li L, et al. Smad3 C-terminal phosphorylation site mutation attenuates the hepatoprotective effect of salvianolic acid B against hepatocarcinogenesis. Food Chem Toxicol. 2021;147:111912. doi:10.1016/j.fct.2020.111912

42. Su H, Ma Z, Guo A, Wu H, Yang X. Salvianolic acid B protects against sepsis-induced liver injury via activation of SIRT1/PGC-1 $\alpha$ signaling. Exp Ther Med. 2020;20(3):2675-2683. doi:10.3892/ etm.2020.9020

43. Yan XF, Zhao P, Ma DY, et al. Salvianolic acid B protects hepatocytes from $\mathrm{H}_{2} \mathrm{O}_{2}$ injury by stabilizing the lysosomal membrane. World J Gastroenterol. 2017;23(29):5333-5344. doi:10.3748/wjg. v23.i29.5333

44. Li C, Li Q, Liu YY, et al. Protective effects of notoginsenoside R1 on intestinal ischemia-reperfusion injury in rats. $\mathrm{Am} J$ Physiol Gastrointest Liver Physiol. 2014;306(2):G111-G122. doi:10.1152/ ajpgi.00123.2013

45. Song B, Sun Y, Chu Y, et al. Ginsenoside Rb1 alleviated high-fatdiet-induced hepatocytic apoptosis via peroxisome proliferator-activated receptor $\gamma$. Biomed Res Int. 2020;2020:2315230. doi:10.1155/ 2020/2315230

46. Gao X, Xu J, Liu H. Protective effects of catalpol on mitochondria of hepatocytes in cholestatic liver injury. Mol Med Rep. 2020;22 (3):2424-2432. doi:10.3892/mmr.2020.11337

47. Liu L, Gao H, Wang H, et al. Catalpol promotes cellular apoptosis in human HCT116 colorectal cancer cells via microRNA-200 and the downregulation of PI3K-Akt signaling pathway. Oncol Lett. 2017;14 (3):3741-3747. doi:10.3892/ol.2017.6580

48. Kim H, Pan JH, Kim SH, Lee JH, Park JW. Chlorogenic acid ameliorates alcohol-induced liver injuries through scavenging reactive oxygen species. Biochimie. 2018;150:131-138. doi:10.1016/j. biochi.2018.05.008

49. Zhong S, Li YG, Ji DF, Lin TB, Lv ZQ. Protocatechualdehyde induces S-phase arrest and apoptosis by stimulating the p27 (KIP1)-Cyclin A/D1-CDK2 and mitochondrial apoptotic pathways in HT-29 cells. Molecules. 2016;21(7):934. doi:10.3390/ molecules21070934

50. Zhang X, Ding J, Gou C, et al. Qingchangligan formula attenuates the inflammatory response to protect the liver from acute failure induced by D-galactosamine/ Lipopolysaccharide in mice. J Ethnopharmacol. 2017;201:108-116. doi:10.1016/j.jep.2016.11.007
51. Moreau R. The pathogenesis of ACLF: the inflammatory response and immune function. Semin Liver Dis. 2016;36(2):133-140. doi:10.1055/s-0036-1583199

52. Triantafyllou E, Woollard KJ, McPhail MJW, Antoniades CG, Possamai LA. The role of monocytes and macrophages in acute and acute-on-chronic liver failure. Front Immunol. 2018;9:2948. doi:10.3389/fimmu.2018.02948

53. Blasco-Algora S, Masegosa-Ataz J, Gutiérrez-García ML, AlonsoLópez S, Fernández-Rodríguez CM. Acute-on-chronic liver failure: pathogenesis, prognostic factors and management. World $J$ Gastroenterol. 2015;21(42):12125-12140. doi:10.3748/wjg.v21. i42.12125

54. Haderski GJ, Kandar BM, Brackett CM, et al. TLR5 agonist entolimod reduces the adverse toxicity of TNF while preserving its antitumor effects. PLoS One. 2020;15(2):e0227940. doi:10.1371/journal. pone. 0227940

55. Wang X, Wu L, Zhang Q, et al. Methyl 3,4-dihydroxybenzoate protects against D-galN/LPS-induced acute liver injury by inhibiting inflammation and apoptosis in mice. J Pharm Pharmacol. 2019;71 (7):1082-1088. doi:10.1111/jphp.13091

56. Takheaw N, Earwong P, Laopajon W, Pata S, Kasinrerk W, Kanellopoulos J. Interaction of CD99 and its ligand upregulates IL6 and TNF- $\alpha$ upon T cell activation. PLoS One. 2019;14(5): e0217393. doi:10.1371/journal.pone.0217393

57. Ribeiro CV, Rocha BFB, Oliveira E, et al. Leishmania infantum induces high phagocytic capacity and intracellular nitric oxide production by human proinflammatory monocyte. Mem Inst Oswaldo Cruz. 2020;115:e190408. doi:10.1590/0074-02760190408

58. Zhang Z, Liu W, Zhao L, et al. Retinoblastoma 1 protects $\mathrm{T}$ cell maturation from premature apoptosis by inhibiting E2F1. Development. 2018;145(1):dev158139. doi:10.1242/dev.158139

59. Gao G, Yu Z, Yan J, et al. Lowering blood ammonia prevents hepatocyte injury and apoptosis. Int J Clin Exp Med. 2015;8 (8):12347-12355.

60. Taminiau A, Draime A, Tys J, et al. HOXA1 binds RBCK1/HOIL-1 and TRAF2 and modulates the TNF/NF- $\kappa \mathrm{B}$ pathway in a transcription-independent manner. Nucleic Acids Res. 2016;44 (15):7331-7349. doi:10.1093/nar/gkw606

61. Bierbrauer A, Jacob M, Vogler M, Fulda S. A direct comparison of selective BH3-mimetics reveals BCL-XL, BCL-2 and MCL-1 as promising therapeutic targets in neuroblastoma. $\mathrm{Br} J$ Cancer. 2020;122(10):1544-1551. doi:10.1038/s41416-020-0795-9

\section{Publish your work in this journal}

Drug Design, Development and Therapy is an international, peerreviewed open-access journal that spans the spectrum of drug design and development through to clinical applications. Clinical outcomes, patient safety, and programs for the development and effective, safe, and sustained use of medicines are a feature of the journal, which has also been accepted for indexing on PubMed Central. The manuscript management system is completely online and includes a very quick and fair peer-review system, which is all easy to use. Visit http://www. dovepress.com/testimonials.php to read real quotes from published authors. 